\title{
29. CHEMICAL ANALYSES OF RED SEA SEDIMENTS
}

Frank T. Manheim, U. S. Geological Survey, Woods Hole, Massachusetts David E. Siems, U. S. Geological Survey, Denver, Colorado

One of the prime motives for exploring the deeper subsurface sediments of the Red Sea floor was to gain information on the geochemical systems controlling the hot brine-metalliferous deposits. Accordingly, a strong effort was made to provide both shipboard and laboratory means of analysis of the recovered phases. Shipboard spectrographic analyses (semiquantitative) provided both detailed surveys of chemical properties of the cores and the ability to analyze small subsamples and separated particles such as pyrite, sphalerite, organisms, and similar minute objects. Over 5000 such analyses are provided in Tables 1 to 5 . Some supplementary data provided by subsequent analyses for $\mathrm{K}, \mathrm{Zn}$, and $\mathrm{Ag}$ in the Denver field laboratories of the U. S. Geological Survey have been added to the tables.
A second body of data is provided in Table 6, which gives quantitative data on major and minor constituents, performed by the Washington analytical laboratories of the U.S. Geological Survey. A third group of analyses are partial analyses on evaporitic rocks (anhydrite and rock salt) (Table 18, in Manheim Chapter 38, this volume).

In addition to the above, a number of the collaborating laboratories are providing more specific chemical data on special phases and constituents of the Red Sea materials. Most of these data are included in chapters in the Geochemical section of this volume.

The significance of the data is discussed in Manheim (Chapter 38 , this volume) and in the respective reports of shipboard workers and cooperating share laboratories. 
TABLE 1A

Semiquantitative Spectrographic Analyses of Samples from Site 225

\begin{tabular}{|c|c|c|c|c|c|c|c|c|c|c|c|c|c|c|c|c|c|c|c|c|c|c|c|c|c|c|}
\hline \multirow[b]{2}{*}{ Sample } & \multirow{2}{*}{$\begin{array}{c}\text { Depth } \\
(\mathrm{m})\end{array}$} & \multicolumn{8}{|c|}{ Major Elements (\%) } & \multicolumn{16}{|c|}{ Minor and Trace Elements (ppm) } & \multirow[b]{2}{*}{ Description } \\
\hline & & $\mathrm{Si}$ & $\mathrm{K}$ & $\mathrm{Al}$ & $\mathrm{Fe}$ & $\mathrm{Ti}$ & $\mathrm{Ca}$ & $\mathrm{Mg}$ & $\mathrm{Mn}$ & $\mathrm{Pb}$ & $\mathrm{Ag}$ & $\mathrm{Cu}$ & $\mathrm{Zn}$ & Sn & $\mathrm{Cd}$ & Mo & $\mathrm{Ni}$ & Co & $\mathrm{V}$ & $\mathrm{Ba}$ & Sr & B & As & $\mathrm{Zr}_{\mathrm{r}}$ & $\mathrm{Cr}$ & \\
\hline $1-2,100-101$ & 2.5 & 10 & $0.6^{*}$ & 4 & $15 \mathrm{c}$ & 0.3 & 15 & 3 & 0.1 & 10 & $\mathrm{~N}$ & 10 & $60^{*}$ & $\mathrm{~N}$ & $\mathrm{~N}$ & $\mathrm{~N}$ & 70 & 7 & 20 & 50 & 500 & 20 & $\mathrm{~N}$ & 70 & 70 & Yw brn soupy carb mud \\
\hline & 3.6 & 5 & & 2.5 & $0.5 \mathrm{C}$ & 0.2 & 201 & 1.5 & 0.01 & $k 10$ & $\mathrm{~N}$ & 20 & $\mathrm{~N}$ & $\mathrm{~N}$ & $\mathrm{~N}$ & $\mathrm{~N}$ & 15 & 10 & 20 & $\mathrm{~N}>$ & $>5000$ & 30 & $\mathrm{~N}$ & 15 & 20 & $\begin{array}{l}\text { Yw brn lithified } \\
\text { limestone crust }\end{array}$ \\
\hline $1-4,70-73$ & 5.2 & 10 & & 4 & $0.7 \mathrm{C}$ & 0.2 & 20 & 1.5 & 0.02 & $\leq 10$ & Na & 5 & $\mathrm{~N}$ & $\mathbf{N}$ & $\mathrm{N}$ & $\mathrm{N}$ & 10 & 5 & 15 & $\mathrm{~N}>$ & $>5000$ & 10 & $\mathrm{~N}$ & 15 & 50 & $\begin{array}{l}\text { Yw brn cream wh } \\
\text { limestone and (kidney-shaped } \\
\text { chert not analyzed) }\end{array}$ \\
\hline $1-4,105$ & 5.5 & 5 & $0.3^{*}$ & 2.5 & $0.5 \mathrm{C}$ & 0.15 & 20 & 1 & 0.01 & $\leq 10$ & $\mathrm{~N}$ & 5 & $40^{*}$ & $\mathrm{~N}$ & $\mathrm{~N}$ & $\mathrm{~N}$ & 7 & $\leq 5$ & 10 & $\mathrm{~N}>$ & $>5000$ & 10 & $\mathrm{~N}$ & 20 & 20 & $\begin{array}{l}\text { Yw brn lithified } \\
\text { limestone crust }\end{array}$ \\
\hline $1-5,85$ & 6.8 & 10 & & 4 & $0.5 c$ & $0.2>$ & 20 & 1.5 & 0.01 & $\leq 10$ & $\mathrm{~N}$ & 5 & $\mathrm{~N}$ & $\mathrm{~N}$ & $\mathrm{~N}$ & $\mathrm{~N}$ & 10 & $\leq 5$ & 10 & $N>$ & $>5000$ & 10 & $\mathrm{~N}$ & 20 & 70 & $\begin{array}{l}\text { Yw brn lithified } \\
\text { limestone crust }\end{array}$ \\
\hline $1-6,10$ & 7.6 & 10 & & 4 & 0.50 & $0.2>$ & 20 & 1.5 & 0.015 & $\leq 10$ & $\mathrm{~N}$ & 5 & $80^{*}$ & $\mathrm{~N}$ & N & $\mathrm{N}$ & 50 & 10 & 20 & $\mathrm{~N}>$ & $>5000$ & 10 & $N$ & 20 & 50 & Gy grn lithified limestone \\
\hline $3-3,100-101$ & 20.5 & 12 & & 5 & 30 & 0.3 & 20 & 5 & 0.2 & 10 & $\mathrm{~N}$ & 20 & $N$ & $\mathbf{N}$ & N & $\mathrm{N}$ & 50 & 10 & 30 & 70 & 100 & 10 & $\mathrm{~N}$ & 50 & 100 & Yw gy grn carb mud \\
\hline $3-3,102-103$ & 22.0 & 12 & & 5 & 5 & 0.5 & 20 & 5 & 0.2 & $\leq 10$ & $\mathrm{~N}$ & 20 & $\mathrm{~N}$ & $\mathrm{~N}$ & $\mathrm{~N}$ & $\mathrm{~N}$ & 50 & 10 & 30 & 100 & 1000 & 10 & $\leq 10$ & 70 & 100 & Gy grn carb mud \\
\hline $3-4,0-10$ & 22.5 & $<2$ & & $0.3>2$ & & 0.005 & 1.5 & 0.02 & 0.07 & $\mathrm{~N}$ & $\mathrm{~N}$ & 100 & $\mathrm{~N}$ & $\mathrm{~N}$ & $\mathrm{~N}$ & 300 & 500 & 100 & $\leq 10$ & $\mathrm{~N}$ & $\leq 100$ & 20 & 3000 & 10 & $\mathrm{~N}$ & $\begin{array}{l}\text { Slv wh pyrite "branch" } \\
\text { concretion: also, } 500 \mathrm{ppm} \mathrm{Sb}\end{array}$ \\
\hline $4-3,60-61$ & 26.6 & 12 & & 0.5 & 2 & 0.3 & 20 & 5 & 0.2 & 10 & $\mathrm{~N}$ & 10 & $\mathrm{~N}$ & $\mathrm{~N}$ & $\mathrm{~N}$ & $\mathrm{~N}$ & 70 & 10 & 20 & 20 & 1000 & 50 & $\mathrm{~N}$ & 50 & 10 & Lt gy grn carb mud \\
\hline $4-4,90-91$ & 28.9 & 15 & & 5 & 3 & 0.3 & 10 & 5 & 0.3 & 10 & $\mathrm{~N}$ & 50 & $\mathrm{~N}$ & $\mathrm{~N}$ & $\mathrm{~N}$ & $\mathrm{~N}$ & 20 & 10 & 20 & 70 & 1000 & 20 & $\mathrm{~N}$ & 70 & 100 & Gy grn brn carb mud \\
\hline $4-5,80-81$ & 29.8 & 10 & & $>5$ & 1 & 0.2 & 20 & 1.5 & 0.1 & 10 & 0.5 & 100 & $\left\{\begin{array}{l}200^{*} \\
200\end{array}\right.$ & $\mathbf{N}$ & $\mathrm{N}$ & 200 & 200 & 20 & 700 & 1000 & 1500 & 20 & $\mathrm{~N}$ & 20 & 70 & Dk grn bk pyrite mud \\
\hline $45,110-111$ & 30.1 & 15 & & $>5$ & 5 & 0.3 & 20 & 7 & 0.15 & $\leq 10$ & N & 70 & N & $\mathrm{N}$ & $\mathrm{N}$ & 5 & 150 & 20 & 50 & 100 & 1000 & 20 & $\mathrm{~N}$ & 50 & 10 & Lt gy grn carb mud \\
\hline $4-6,30-31$ & 30.8 & 10 & & 5 & 50 & 0.3 & 15 & 2 & 0.2 & 10 & $\mathrm{~N}$ & 50 & 80 & $\mathrm{~N}$ & $\mathrm{~N}$ & 30 & 50 & 50 & 300 & 300 & 1000 & 20 & 15 & 30 & 100 & $\begin{array}{l}\text { Dk grn brn clay and } \\
\text { pyrite rich mud }\end{array}$ \\
\hline $5-1,85-86$ & 27.9 & 12 & & 5 & 5 & 0.3 & 15 & 2 & 0.15 & 10 & $\mathrm{~N}$ & 70 & $\mathrm{~N}$ & $\mathrm{~N}$ & $\mathrm{~N}$ & 50 & 100 & 20 & 500 & 300 & 1000 & 20 & $\mathrm{~N}$ & 30 & 100 & $\begin{array}{l}\text { Dk grn bk clay and } \\
\text { pyrite-rich mud }\end{array}$ \\
\hline $5-2,80-81$ & 29.3 & 12 & & 5 & 5 & 0.3 & 20 & 5 & 0.2 & 10 & $\mathrm{~N}$ & 50 & $\mathrm{~N}$ & $\mathrm{~N}$ & $\mathrm{~N}$ & 5 & 100 & 20 & 50 & 100 & 1000 & 20 & $\mathbf{N}$ & 50 & 100 & Gy grn calcareous mud \\
\hline
\end{tabular}


Table 1A - Continued

\begin{tabular}{|c|c|c|c|c|c|c|c|c|c|c|c|c|c|c|c|c|c|c|c|c|c|c|c|c|c|c|}
\hline \multirow[b]{2}{*}{ Sample } & \multirow{2}{*}{$\begin{array}{c}\text { Depth } \\
(\mathrm{m})\end{array}$} & \multicolumn{8}{|c|}{ Major Elements (\%) } & \multicolumn{16}{|c|}{ Minor and Trace Elements (ppm) } & \multirow[b]{2}{*}{ Description } \\
\hline & & $\mathrm{Si}$ & $\mathbf{K}$ & Al & $\mathrm{Fe}$ & $\mathrm{Ti}$ & $\mathrm{Ca}$ & $\mathrm{Mg}$ & $\mathrm{Mn}$ & $\mathrm{Pb}$ & $\mathrm{Ag}$ & $\mathrm{Cu}$ & $\mathrm{Zn}$ & Sn & $\mathrm{Cd}$ & Mo & $\mathrm{Ni}$ & Co & V & $\mathrm{Ba}$ & $\mathrm{Sr}$ & B & As & $\mathrm{Zr}$ & $\mathrm{Cr}$ & \\
\hline $6-1,130-133$ & 37.2 & 10 & & $>5$ & 1 & $0.2>2$ & 20 & 5 & 0.1 & $<10$ & $\mathrm{~N}$ & 5 & $\mathrm{~N}$ & $\mathrm{~N}$ & $\mathrm{~N}$ & $\mathrm{~N}$ & 20 & 7 & 20 & $\mathrm{~N}$ & 3000 & 10 & $\mathrm{~N}$ & 30 & $\mathrm{~N}$ & $\begin{array}{l}\text { Cream to brn lithified } \\
\text { rounded limestone grains }\end{array}$ \\
\hline $8-3,52-53$ & 48.5 & 15 & & $>5$ & 5 & 0.3 & 205 & 5 & 0.5 & 10 & $\mathbf{N}$ & 50 & $\mathrm{~N}$ & $\mathrm{~N}$ & $\mathrm{~N}$ & $\mathrm{~N}$ & 100 & 20 & 30 & 100 & 1000 & 20 & $\mathrm{~N}$ & 50 & 150 & Gy brn pasty carb mud \\
\hline $8, \mathrm{CC}$ & 54 & $\mathrm{~N}$ & & $\mathrm{~N}$ & $\mathrm{~N}$ & $\mathbf{N}$ & 0.11 & $\mathrm{~N}$ & $\mathrm{~N}$ & $N$ & $N$ & $\mathrm{~N}$ & $\mathrm{~N}$ & $\mathrm{~N}$ & $\mathrm{~N}$ & $N$ & $\mathrm{~N}$ & $\mathrm{~N}$ & 100 & $\mathrm{~N}$ & $\mathrm{~N}$ & $\mathrm{~N}$ & $\mathrm{~N}$ & $\mathrm{~N}$ & $\mathrm{~N}$ & $\begin{array}{l}\text { Amber "glassy" fragments } \\
\text { also } 70 \mathrm{ppm} \mathrm{B} 1\end{array}$ \\
\hline $9-3,75-77$ & 57.8 & 15 & & $>5$ & 5 & 0.5 & 20 & 5 & 0.3 & 10 & $\mathrm{~N}$ & 20 & $\mathrm{~N}$ & $\mathrm{~N}$ & $\mathrm{~N}$ & $\mathrm{~N}$ & 70 & 20 & 20 & 70 & 500 & 10 & $\mathrm{~N}$ & 50 & 100 & Lt gy grn pyrite mud \\
\hline $9-6,90-92$ & 62.4 & 15 & & 5 & 5 & 0.32 & 20 & 2 & 0.1 & 10 & $\mathrm{~N}$ & 100 & $150^{*}$ & $\mathrm{~N}$ & $\mathrm{~N}$ & 300 & 150 & 20 & 1000 & 1500 & 1000 & 30 & 10 & 30 & 100 & Dk brn bk pyrite mud \\
\hline $9-6,112-114$ & 62.5 & 15 & & $>5$ & 3 & 0.52 & 20 & 5 & 0.3 & $<10$ & $\mathrm{~N}$ & 20 & $\mathrm{~N}$ & $\mathrm{~N}$ & $\mathrm{~N}$ & $\mathrm{~N}$ & 100 & 15 & 20 & 100 & 1000 & 10 & $\mathrm{~N}$ & 70 & 100 & Lt grn gy carb mud \\
\hline $9-6,118-120$ & 62.6 & 15 & & $>5$ & 2 & $0.3>2$ & 205 & 5 & 0.3 & $<10$ & $\mathrm{~N}$ & 15 & $\mathrm{~N}$ & $\mathrm{~N}$ & $\mathrm{~N}$ & $\mathrm{~N}$ & 50 & 7 & 20 & 50 & 1000 & 10 & $\mathrm{~N}$ & 50 & 150 & Lt grn gy carb mud \\
\hline $9, \mathrm{CC}$ & 63 & $\mathrm{~N}$ & & $<0.3$ & $>20$ & $\mathrm{~N}$ & $1.5<0$ & $<0.03$ & 0.15 & $\mathrm{~N}$ & $\mathrm{~N}$ & 300 & $\mathrm{~N}$ & $\mathrm{~N}$ & $\mathrm{~N}$ & 20 & 500 & 150 & $<15$ & $\mathrm{~N}$ & $\mathrm{~N}$ & $\mathrm{~N}$ & $\mathrm{~N}$ & $\mathrm{~N}$ & $\mathrm{~N}$ & Small pyrite fragments \\
\hline $10-2,104-105$ & 65.5 & 12 & & 5 & 3 & 0.52 & 202 & 2 & 0.2 & $<10$ & $\mathrm{~N}$ & 20 & $\mathrm{~N}$ & $\mathrm{~N}$ & $\mathrm{~N}$ & $\mathrm{~N}$ & 50 & 10 & 30 & 100 & 700 & 10 & $\mathrm{~N}$ & 70 & 100 & Gy brn gritty carb mud \\
\hline $11, \mathrm{CC}$ & 77 & 7 & & 1.5 & $>20$ & 0.01 & 1.50 & 0.02 & 0.007 & N & $\mathrm{N}$ & 70 & $\mathrm{~N}$ & $\mathrm{~N}$ & $\mathrm{~N}$ & 500 & 10 & 5 & 5 & $\mathrm{~N}$ & $\mathrm{~N}$ & 20 & $\mathrm{~N}$ & $\mathrm{~N}$ & $\mathrm{~N}$ & $\begin{array}{l}\text { Pyrite; flat plate } 2 \times 3 \mathrm{~mm} \\
\text { consisting of minute crystalite }\end{array}$ \\
\hline $12-1,115-116$ & 78.1 & 12 & & 4 & 5 & 0.3 & 20 & 5 & 0.2 & $<10$ & $\mathrm{~N}$ & 100 & $\mathrm{~N}$ & $\mathrm{~N}$ & $\mathrm{~N}$ & 15 & 70 & 15 & 70 & 150 & 1000 & 10 & $\mathrm{~N}$ & 70 & 70 & Gritty olive mud; soupy \\
\hline $13-6,0-10$ & 84.5 & 7 & & 5 & 0.3 & 0.15 & 20 & 2 & 0.015 & $<10$ & $\mathrm{~N}$ & 5 & $\mathrm{~N}$ & $\mathrm{~N}$ & $\mathrm{~N}$ & $\mathrm{~N}$ & 5 & $\mathrm{~N}$ & 10 & $\mathrm{~N}>$ & $>5000$ & 10 & $\mathrm{~N}$ & 15 & 150 & Yw lithified crust \\
\hline $13-6,45-46$ & 85 & 12 & & 5 & 5 & 0.5 & 20 & 5 & 0.3 & $<10$ & $\mathrm{~N}$ & 10 & $\mathrm{~N}$ & $\mathrm{~N}$ & $\mathrm{~N}$ & $\mathrm{~N}$ & 70 & 15 & 30 & 50 & 1000 & 10 & $\mathrm{~N}$ & 70 & 70 & Lt gy grn carb mud \\
\hline $14-2,30-31$ & 87.8 & 15 & & 5 & 3 & 0.3 & 20 & 5 & 0.2 & 10 & $\mathrm{~N}$ & 100 & $\mathrm{~N}$ & $\mathrm{~N}$ & $\mathrm{~N}$ & $\mathrm{~N}$ & 100 & 20 & 30 & 50 & 700 & 10 & $\mathrm{~N}$ & 50 & 100 & Gy grn carb mud \\
\hline 142,80 & 88.4 & 10 & & 3 & $=20$ & 0.2 & 7 & 0.3 & 0.015 & $\mathrm{~N}$ & $\mathrm{~N}$ & 30 & $\mathrm{~N}$ & $\mathrm{~N}$ & $\mathrm{~N}$ & 20 & 150 & 50 & 10 & $\mathrm{~N}$ & 150 & 10 & $\mathrm{~N}$ & 10 & 20 & Pyrite concretions \\
\hline $14-2,125-126$ & 88.8 & 10 & & 4 & 3 & 0.3 & 20 & 1.5 & 0.1 & 10 & $\mathrm{~N}$ & 50 & $50 *$ & $\mathrm{~N}$ & $\mathrm{~N}$ & 150 & 70 & 15 & 700 & 300 & 1000 & 10 & 10 & 20 & 50 & Dk olive pyrite mud \\
\hline $14-2,141-142$ & 88.9 & 12 & & 4 & 5 & 0.3 & 20 & 5 & 0.2 & $\leq 10$ & $\mathrm{~N}$ & 10 & $\mathrm{~N}$ & $\mathrm{~N}$ & $\mathrm{~N}$ & 20 & 70 & 15 & 30 & 50 & 1000 & 10 & $\mathrm{~N}$ & 20 & 70 & Gy carb mud \\
\hline $14-3,39-40$ & 89.4 & 15 & & 5 & 5 & 0.5 & 20 & 7 & 0.2 & 10 & $\mathrm{~N}$ & 70 & $\mathrm{~N}$ & $\mathrm{~N}$ & $\mathrm{~N}$ & $\mathrm{~N}$ & 100 & 15 & 50 & 70 & 1000 & 30 & $\mathrm{~N}$ & 50 & 00 & Med gy carb mud \\
\hline $14-3,80-81$ & 89.8 & 12 & & 5 & 3 & 0.2 & 20 & 1.5 & 0.2 & 10 & $\mathrm{~N}$ & 20 & $46^{*}$ & $\mathrm{~N}$ & $\mathrm{~N}$ & 30 & 70 & 10 & 500 & 700 & 1000 & 20 & $\mathrm{~N}$ & 15 & 70 & $\begin{array}{l}\text { Dk brownish to olive } \\
\text { pyrite mud }\end{array}$ \\
\hline $14-4,59-60$ & 91.1 & 12 & & 5 & 3 & 0.3 & 20 & 3 & 0.5 & 10 & $\mathrm{~N}$ & 10 & $\mathrm{~N}$ & $\mathrm{~N}$ & $\mathrm{~N}$ & $\mathrm{~N}$ & 50 & 10 & 20 & 50 & 700 & 10 & $\mathrm{~N}$ & 20 & 70 & Lt cream gy grn carb mud \\
\hline $14-4,109-110$ & 91.6 & 12 & & 5 & 5 & 0.3 & 20 & 2 & 0.15 & $\leq 10$ & $\mathrm{~N}$ & 50 & $65^{*}$ & $\mathrm{~N}$ & $\mathrm{~N}$ & 200 & 100 & 20 & 700 & 700 & 1000 & 30 & $\mathrm{~N}$ & 20 & 70 & Dk brn bk pyritic mud \\
\hline $14, \mathrm{CC}$ & 95 & 5 & & 0.7 & 20 & 0.005 & 2 & 0.07 & 0.15 & $\mathrm{~N}$ & $\mathrm{~N}$ & 150 & $\mathrm{~N}$ & $\mathrm{~N}$ & $\mathrm{~N}$ & 50 & 500 & 150 & 20 & $\mathrm{~N}$ & $\mathrm{~N} \leq$ & $\leq 20$ & $\mathrm{~N}$ & $\mathrm{~N}$ & 200 & $\begin{array}{l}\text { Pyrite; small tube } \\
\text { fillings plus organic } \\
\text { replacements }\end{array}$ \\
\hline $15-2,133-134$ & 97.8 & 12 & & 4 & 3 & $1.3>$ & 20 & 3 & 0.2 & $\leq 10$ & $\mathrm{~N}$ & 5 & $\mathrm{~N}$ & $\mathrm{~N}$ & $\mathrm{~N}$ & $\mathrm{~N}$ & 15 & 7 & 20 & 50 & 1000 & 10 & $\mathrm{~N}$ & 20 & 50 & Lt buff carb mud \\
\hline $16-3,98-99$ & 108 & 10 & & 4 & 3 & 0.2 & 15 & 5 & 0.2 & $<10$ & $\mathrm{~N}$ & 30 & $\mathrm{~N}$ & $\mathrm{~N}$ & $\mathrm{~N}$ & $\mathrm{~N}$ & 20 & 7 & 20 & 50 & 300 & 10 & $\mathrm{~N}$ & 15 & 70 & Lt gy brn carb mud \\
\hline $16, \mathrm{CC}$ & 113 & $\leq 2$ & & $\leq 6 \leq$ & $\leq 0.005$ & $\mathrm{~N}>$ & 20 & 0.2 & 0.05 & $\mathrm{~N}$ & $\mathrm{~N}$ & $\mathrm{~N}$ & $\mathrm{~N}$ & $\mathrm{~N}$ & $\mathrm{~N}$ & $\mathrm{~N}$ & $\mathrm{~N}$ & $\mathrm{~N}$ & $<200$ & $\mathrm{~N}$ & $\mathrm{~N}$ & $\mathrm{~N}$ & $\mathrm{~N}$ & $\mathrm{~N}$ & $\mathrm{~N}$ & $\begin{array}{l}\text { Planktonic forams } \\
\text { "fresh" looking }\end{array}$ \\
\hline $16, \mathrm{CC}$ & 113 & 5 & & 7 & 0.1 & $10.01>$ & & 0.07 & 0.3 & $\leq 20$ & $\mathrm{~N}$ & 200 & $\mathrm{~N}$ & $\mathrm{~N}$ & $\mathrm{~N}$ & $\mathrm{~N}$ & $\leq 20$ & $\mathrm{~N}$ & 20 & $\leq 50$ & 300 & $\mathrm{~N}$ & $\mathrm{~N}$ & 20 & 200 & $\begin{array}{l}\text { Sugary recrystallized } \\
\text { organisms and some } \\
\text { echinoderm fragments; } \\
\text { also, trace of } \mathrm{Bi}<20 \mathrm{ppm}\end{array}$ \\
\hline $17-2,61-61$ & 115.1 & 12 & & 5 & 3 & 0.3 & 20 & 5 & 0.2 & $\mathrm{~N}$ & $\mathrm{~N}$ & 15 & $\mathrm{~N}$ & $\mathrm{~N}$ & $\mathrm{~N}$ & $\mathrm{~N}$ & 20 & 7 & 20 & 50 & 500 & 10 & $\mathrm{~N}$ & 50 & 70 & Lt gy brn carb mud \\
\hline $18-1,82-83$ & 122.8 & 15 & & $\geq 5$ & 7 & 0.5 & 15 & 5 & 0.15 & 10 & $\mathrm{~N}$ & 50 & $\mathrm{~N}$ & $\mathrm{~N}$ & $\mathrm{~N}$ & $\mathrm{~N}$ & 100 & 20 & 50 & 100 & 700 & 30 & $\mathrm{~N}$ & 50 & 100 & Gy carb mud \\
\hline $18-3,55-56$ & 125.5 & 7 & & 5 & 7 & 0.3 & 20 & 1.5 & 0.2 & 10 & $\mathrm{~N}$ & 70 & $\mathrm{~N}$ & $\mathrm{~N}$ & $\mathrm{~N}$ & 100 & 100 & 20 & 500 & 500 & 700 & 10 & $\mathrm{~N}$ & 30 & 70 & Dk brn bk pyrite mud \\
\hline $18-3,119-120$ & 126.2 & 15 & & 5 & 5 & 0.3 & 15 & 2 & 0.2 & 10 & $\mathrm{~N}$ & $70<$ & $<200$ & $\mathrm{~N}$ & $\mathrm{~N}$ & $\mathrm{~N}$ & 100 & 50 & 50 & 100 & 700 & 20 & $\mathrm{~N}$ & 50 & 100 & Medium gy carb mud \\
\hline $18-4,30-31$ & 126.8 & 15 & & 5 & 5 & 0.5 & 15 & 2 & 0.2 & $\leq 10$ & $\mathrm{~N}$ & 70 & $\mathrm{~N}$ & $\mathrm{~N}$ & $\mathrm{~N}$ & $\mathrm{~N}$ & 100 & 20 & 50 & 100 & 500 & 20 & $\mathrm{~N}$ & 70 & 100 & Medium gy carb mud \\
\hline
\end{tabular}


TABLE 1A - Continued

\begin{tabular}{|c|c|c|c|c|c|c|c|c|c|c|c|c|c|c|c|c|c|c|c|c|c|c|c|c|c|c|}
\hline \multirow[b]{2}{*}{ Sample } & \multirow{2}{*}{$\begin{array}{c}\text { Depth } \\
(\mathrm{m})\end{array}$} & \multicolumn{8}{|c|}{ Major Elements (\%) } & \multicolumn{16}{|c|}{ Minor and Trace Elements (ppm) } & \multirow[b]{2}{*}{ Description } \\
\hline & & Si & $\mathrm{K}$ & $\mathrm{Al}$ & $\mathrm{Fe}$ & $\mathrm{Ti}$ & $\mathrm{Ca}$ & $\mathrm{Mg}$ & $\mathrm{Mn}$ & $\mathrm{Pb}$ & $\mathrm{Ag}$ & $\mathrm{Cu}$ & $\mathrm{Zn}$ & Sn & $\mathrm{Cd}$ & Mo & $\mathrm{Ni}$ & Co & V & $\mathrm{Ba}$ & $\mathrm{Sr}$ & B & As & $\mathrm{Zr}$ & $\mathrm{Cr}$ & \\
\hline $18-4,70-71$ & 127.2 & 12 & & 5 & 5 & 0.2 & 20 & 1.5 & 0.2 & 10 & $\mathrm{~N}$ & 100 & $50 *$ & $\mathrm{~N}$ & $\mathrm{~N}$ & 20 & 70 & 20 & 200 & 700 & 1000 & 10 & $\mathrm{~N}$ & 20 & 70 & Dk gin bk carb mud \\
\hline $22-3,77-79$ & 161.8 & 20 & & 5 & 5 & 0.5 & 10 & 2 & 0.15 & $\mathrm{~N}$ & $\mathrm{~N}$ & 100 & $\mathrm{~N}$ & $\mathrm{~N}$ & $\mathrm{~N}$ & $\mathrm{~N}$ & 150 & 50 & 70 & 150 & 500 & 30 & $\mathrm{~N}$ & 70 & 150 & $\begin{array}{l}\text { Lt gy grn firm mud } \\
\text { Pyrite; yellowish brassy }\end{array}$ \\
\hline $22-6,126-127$ & 166.8 & 7 & & 2 & 20 & 0.015 & $\begin{array}{ll}5 & 0.7\end{array}$ & 70.02 & 0.005 & 50 & $\mathrm{~N}$ & 200 & $\mathrm{~N}$ & $\mathrm{~N}$ & $\mathrm{~N}$ & 200 & 500 & 20 & 10 & $\mathrm{~N}$ & 100 & 20 & $\mathrm{~N}$ & 10 & $\mathrm{~N}$ & fragments \\
\hline $22, C C$ & 167 & 15 & & 3 & 20 & $0.15>$ & $>20$ & 7 & 0.005 & $\mathrm{~N}$ & $\mathrm{~N}$ & 300 & $\mathrm{~N}$ & $\mathrm{~N}$ & $\mathrm{~N}$ & 15 & 200 & 30 & 15 & $\mathrm{~N}$ & 150 & 15 & $\mathrm{~N}$ & $\mathrm{~N}$ & 20 & Pyrite \\
\hline $22, \mathrm{CC}$ & 167 & 30 & & 5 & 1.5 & $0.07>$ & $>20$ & 7 & 7.5 & $\mathrm{~N}$ & $\mathrm{~N}$ & $\mathrm{~N}$ & $\mathrm{~N}$ & $\mathrm{~N}$ & 100 & $\mathrm{~N}$ & 50 & $\mathrm{~N}$ & 10 & $\mathrm{~N}$ & 500 & $\mathrm{~N}$ & $\mathrm{~N}$ & $\mathrm{~N}$ & $\mathrm{~N}$ & $\begin{array}{l}\text { Sugary dolomite uniform } \\
\text { crystals with some pyrite }\end{array}$ \\
\hline $23-1,102$ & 168 & 15 & & 5 & 7 & 0.7 & 1.5 & 2 & 0.07 & 10 & $\mathbf{N}$ & 200 & $\mathrm{~N}$ & $\mathrm{~N}$ & $\mathrm{~N}$ & $\mathrm{~N}$ & 150 & 50 & 100 & 150 & 100 & 50 & $\mathrm{~N}$ & 50 & 100 & Grn brn bk firm mud \\
\hline $23-2,67$ & 169.2 & 13 & & 3 & 5 & 0.2 & 15 & 10 & 0.5 & 10 & $\mathrm{~N}$ & 100 & $\mathrm{~N}$ & $\mathrm{~N}$ & $\mathrm{~N}$ & $\mathrm{~N}$ & 50 & 10 & 20 & 20 & 150 & 10 & 10 & 20 & 50 & Olive grn dense shale \\
\hline $24-1,60$ & 176.6 & 15 & & 5 & 2 & 0.5 & 10 & 10 & 0.2 & 10 & $\mathrm{~N}$ & 70 & $\mathrm{~N}$ & $\mathrm{~N}$ & $\mathrm{~N}$ & 5 & 50 & 10 & 30 & 100 & 200 & 50 & $\mathrm{~N}$ & 50 & 100 & Gy dolomite limestone \\
\hline $24, \mathrm{CC}$ & 185 & $<2$ & $0.1^{*}$ & 1.5 & 0.15 & 0.007 & 720 & 1 & 0.2 & $\mathrm{~N}$ & $\mathrm{~N}$ & $\mathrm{~N}$ & $\mathrm{~N}$ & $\mathrm{~N}$ & $\mathrm{~N}$ & $\mathrm{~N}$ & $\mathrm{~N}$ & $\mathrm{~N}$ & 10 & $\mathrm{~N}$ & 1000 & 100 & $\mathrm{~N}$ & 20 & 50 & Gy anhydrite rods \\
\hline $25-1,57$ & 185.6 & 25 & & 5 & 7 & 0.7 & 3 & 10 & 0.15 & 10 & $\mathrm{~N}$ & 150 & $170^{*}$ & $\mathrm{~N}$ & $\mathrm{~N}$ & 5 & 100 & 30 & 70 & 150 & 100 & 700 & 10 & 70 & 150 & Bk shale \\
\hline $25-1,102$ & 186 & 15 & $2.5^{*}$ & 5 & 5 & 0.3 & 10 & 10 & 0.2 & 10 & $\mathrm{~N}$ & 70 & $150^{*}$ & $\mathrm{~N}$ & $\mathrm{~N}$ & 5 & 70 & 15 & 70 & 100 & 150 & 300 & $\mathrm{~N}$ & 50 & 100 & $\begin{array}{l}\text { Gy dolomite limestone salt } \\
\text { residue from interstitial wate }\end{array}$ \\
\hline $26-1$ & & N2 & & 0.3 & $\mathrm{~N}$ & 0.003 & 31 & 2 & $\mathrm{~N}$ & $\mathrm{~N}$ & $\mathrm{~N}$ & $\mathrm{~N}$ & $\mathrm{~N}$ & $\mathrm{~N}$ & $\mathrm{~N}$ & $\mathrm{~N}$ & $\mathrm{~N}$ & $\mathrm{~N}$ & 10 & $\mathrm{~N}$ & 150 & 10 & N 1 & $\mathrm{~N}$ & $\mathrm{~N}$ & Dk grn gy shale \\
\hline $\begin{array}{l}26-1 \\
26-1,144\end{array}$ & $\begin{array}{l}194 \\
195.4\end{array}$ & $\begin{array}{l}20 \\
<2\end{array}$ & & $\begin{array}{l}5 \\
1\end{array}$ & $\begin{array}{l}5 \\
0.005\end{array}$ & $\begin{array}{l}0.5 \\
0.002\end{array}$ & $\begin{array}{r}10 \\
220\end{array}$ & $\begin{array}{c}10 \\
0.1\end{array}$ & $\begin{array}{l}0.15 \\
0.001\end{array}$ & $\begin{array}{l}10 \\
N\end{array}$ & $\begin{array}{l}\mathrm{N} \\
\mathrm{N}\end{array}$ & $\begin{array}{l}100 \\
\mathrm{~N}\end{array}$ & $\underset{\mathrm{N}}{\mathrm{N}}$ & $\begin{array}{l}\mathrm{N} \\
\mathrm{N}\end{array}$ & $\begin{array}{l}\mathrm{N} \\
\mathrm{N}\end{array}$ & $\begin{array}{l}\mathrm{N} \\
\mathrm{N}\end{array}$ & $\begin{array}{l}70 \\
\mathrm{~N}\end{array}$ & $\begin{array}{l}20 \\
\mathrm{~N}\end{array}$ & $\begin{array}{l}70 \\
10\end{array}$ & $\begin{array}{l}100 \\
\mathrm{~N}\end{array}$ & $\begin{array}{r}150 \\
3000\end{array}$ & $\begin{array}{r}300 \\
10\end{array}$ & $\begin{array}{l}\mathrm{N} \\
10\end{array}$ & $\begin{array}{l}50 \\
\mathrm{~N}\end{array}$ & $\begin{array}{l}100 \\
\mathrm{~N}\end{array}$ & $\begin{array}{l}\text { Wh-mottled-nodular } \\
\text { anhydrite }\end{array}$ \\
\hline $26-1,60$ & 194.6 & 20 & & 5 & 3 & 0.5 & 2 & 2 & 0.02 & 10 & $\mathrm{~N}$ & 150 & $\{200$ & $\mathrm{N}$ & $\mathrm{N}$ & $\mathrm{N}$ & 70 & 15 & 70 & 100 & 100 & 700 & $\mathrm{~N}$ & 50 & 70 & Bk shale \\
\hline $26-1,107$ & 195 & & $0.1^{*}$ & 1 & 0.005 & 50.002 & 220 & 0.1 & $\mathrm{~N}$ & $\mathrm{~N}$ & $\mathrm{~N}$ & $\mathrm{~N}$ & $20 *$ & & $\mathrm{~N}$ & $\mathrm{~N}$ & $\mathrm{~N}$ & $\mathrm{~N}$ & 10 & $\mathrm{~N}$ & 3000 & 10 & $\mathrm{~N}$ & $\mathrm{~N}$ & $\mathrm{~N}$ & Anhydrite \\
\hline $27-2$ & 203 & N2 & & 0.3 & $\mathrm{~N}$ & $\mathrm{~N}$ & 0.3 & 0.02 & $2 \mathrm{~N}$ & $\mathrm{~N}$ & $\mathrm{~N}$ & $\mathrm{~N}$ & $\mathrm{~N}$ & $\mathrm{~N}$ & $\mathrm{~N}$ & $\mathrm{~N}$ & $\mathrm{~N}$ & $\mathrm{~N}$ & 10 & $\mathrm{~N}$ & $\mathrm{~N}$ & $\mathrm{~N}$ & $\mathrm{~N}$ & $\mathrm{~N}$ & $\mathrm{~N}$ & $\begin{array}{l}\text { Salt crystal from } \\
\text { core liner }\end{array}$ \\
\hline $28-1,90$ & 212.9 & $\mathrm{~N}<$ & $0.1^{*}$ & 1 & 0.05 & 0.003 & 20 & 0.3 & 0.002 & $\mathrm{~N}$ & $\mathrm{~N}$ & $\mathrm{~N}$ & $20 *$ & $\mathrm{~N}$ & $\mathrm{~N}$ & $\mathrm{~N}$ & $\mathrm{~N}$ & $\mathrm{~N}$ & 10 & $\mathrm{~N}$ & 2000 & 10 & $\mathrm{~N}$ & $\mathrm{~N}$ & $\mathrm{~N}$ & Salt layer and anhydrite \\
\hline $28-3,100$ & 216 & $\leqslant 2$ & & 1.5 & & 0.002 & 0.15 & 51.5 & 0.005 & $\mathrm{~N}$ & $\mathrm{~N}$ & 50 & $\mathrm{~N}$ & $\mathrm{~N}$ & $\mathrm{~N}$ & $\mathrm{~N}$ & 50 & $\mathrm{~N}$ & & $\mathrm{~N}$ & 3000 & 10 & $\mathrm{~N}$ & $\mathrm{~N}$ & $\mathrm{~N}$ & $\begin{array}{l}\text { Mixture of anhydrite and } \\
\text { halite }\end{array}$ \\
\hline $29-1,63$ & 221.6 & 25 & & 5 & 5 & 0.2 & 7 & 3 & 0.3 & $\mathrm{~N}$ & $\mathrm{~N}$ & 200 & 100 & $\mathrm{~N}$ & $\mathrm{~N}$ & $\mathrm{~N}$ & 150 & 70 & 70 & 100 & 100 & 20 & $\mathrm{~N}$ & 50 & 100 & Bk shale layer \\
\hline $29-2,86$ & 233.4 & 20 & & $>5$ & 10 & 0.2 & 1 & 10 & 0.5 & $\mathrm{~N}$ & $\mathrm{~N}$ & ${ }^{200}\{$ & $\left\{\begin{array}{l}150^{*} \\
\{200\end{array}\right.$ & $\mathrm{N}$ & $\mathrm{N}$ & $\mathrm{N}$ & 70 & 20 & 150 & 150 & 100 & 50 & $\mathrm{~N}$ & 70 & 50 & Bk shale layer \\
\hline
\end{tabular}

Note:

Analyst David F. Siems. $\mathrm{N}=$ not detected. For list of detection limits see end of Table 1B.

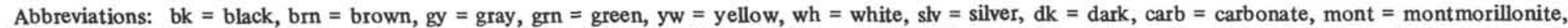

crys $=$ crystal, $\mathbf{l t}=$ light. All samples on air-dry basis (including dried interstitial water). Asterisk indicates supplementary field analysis of $\mathrm{K}, \mathrm{Zn}$, and $\mathrm{Ag}$

by atomic absorption, Denver U.S.G.S. laboratories. 
TABLE 1B

Analysis of Separated Grains, Chiefly Pyrite, from Table 1A

\begin{tabular}{|c|c|c|c|c|c|c|c|c|c|c|c|c|c|c|c|c|c|c|c|c|c|c|c|c|c|c|}
\hline \multirow[b]{2}{*}{ Sample } & \multirow{2}{*}{$\begin{array}{c}\text { Depth } \\
\text { (m) }\end{array}$} & \multicolumn{8}{|c|}{ Major Elements (\%) } & \multicolumn{16}{|c|}{ Minor Elements (ppm) } & \multirow[b]{2}{*}{ Description } \\
\hline & & $\mathrm{Si}$ & $\mathbf{K}$ & $\mathrm{Al}$ & $\mathrm{Fe}$ & $\mathrm{Ti}$ & $\mathrm{Ca}$ & $\mathrm{Mg}$ & Mn & $\mathrm{Pb}$ & $\mathrm{Ag}$ & $\mathrm{Cu}$ & $\mathrm{Zn}$ & $\mathrm{Sn}$ & Cd & Mo & $\mathrm{Ni}$ & Co & $\mathrm{V}$ & $\mathrm{Ba}$ & Sr & B & As & $\mathrm{Zr}$ & $\mathrm{Cr}$ & \\
\hline $3-4,0-10$ & 22.5 & $\leq 2$ & & 0.3 & $>20$ & 0.005 & 1.5 & 0.02 & 0.07 & $\mathrm{~N}$ & $N$ & 100 & $\mathrm{~N}$ & $\mathbf{N}$ & $\mathrm{N}$ & 300 & 500 & 100 & $\leq 10$ & $\mathbf{N}$ & $\leq 100$ & 20 & 3000 & 10 & $\mathrm{~N}$ & $\begin{array}{l}\text { Slv wh pyrite "branch" } \\
\text { concretions: also, } \\
500 \text { ppm Sb }\end{array}$ \\
\hline $9, \mathrm{CC}$ & 63 & $\mathrm{~N}$ & & $\leq 0.3$ & $>20$ & $\mathrm{~N}$ & $1.5 \leq$ & 0.03 & 0.15 & $\mathrm{~N}$ & $\mathrm{~N}$ & 300 & $\mathrm{~N}$ & $\mathrm{~N}$ & $\mathrm{~N}$ & 20 & 500 & 150 & $\leq 15$ & $\mathrm{~N}$ & $\mathrm{~N}$ & $\mathrm{~N}$ & $\mathrm{~N}$ & $\mathrm{~N}$ & $\mathrm{~N}$ & Small pyrite fragments \\
\hline $11, \mathrm{CC}$ & 77 & 7 & & 1.5 & $>20$ & 0.01 & 1.5 & 0.02 & 0.007 & $\mathrm{~N}$ & $\mathrm{~N}$ & 70 & $\mathrm{~N}$ & $\mathrm{~N}$ & $\mathrm{~N}$ & 500 & 10 & 5 & 10 & $\mathrm{~N}$ & $\mathrm{~N}$ & 20 & $\mathrm{~N}$ & $\mathrm{~N}$ & $\mathrm{~N}$ & $\begin{array}{l}\text { Pyrite; flat plate } 2 \times 3 \mathrm{~mm} \\
\text { consisting of minute } \\
\text { crystallites }\end{array}$ \\
\hline $14, C C$ & 95 & 5 & & 0.7 & 20 & 0.005 & 2 & 0.07 & 0.15 & $\mathrm{~N}$ & $\mathbf{N}$ & 150 & $\mathrm{~N}$ & $\mathrm{~N}$ & $\mathrm{~N}$ & 50 & 500 & 150 & 20 & $\mathrm{~N}$ & $\mathrm{~N}:$ & $\leq 20$ & $\mathrm{~N}$ & $\mathrm{~N}$ & 200 & $\begin{array}{l}\text { Pyrite; small tube filling } \\
\text { plus organic replacements }\end{array}$ \\
\hline \multicolumn{2}{|l|}{$22-6,126-127$} & 7 & & 2 & $>20$ & 0.015 & $0.7 \leq$ & 0.02 & 0.005 & 50 & $\mathrm{~N}$ & 200 & $\mathrm{~N}$ & $\mathrm{~N}$ & $\mathrm{~N}$ & 200 & 500 & 20 & $\leq 10$ & $\mathrm{~N}$ & $\leq 100$ & 20 & $\mathrm{~N}$ & $\leq 10$ & $\mathrm{~N}$ & $\begin{array}{l}\text { Pyrite; yellowish "brassy" } \\
\text { fragments }\end{array}$ \\
\hline $22, \mathrm{CC}$ & 167 & 15 & & 3 & $>20$ & $0.15<$ & $<20$ & 7 & 0.005 & $\mathrm{~N}$ & $\mathrm{~N}$ & 300 & $\mathrm{~N}$ & $\mathrm{~N}$ & $\mathrm{~N}$ & 15 & 200 & 30 & 15 & $\mathrm{~N}$ & 150 & 15 & $\mathrm{~N}$ & $\mathrm{~N}$ & 20 & Pyrite \\
\hline 22, CC & 167 & 30 & & 5 & 1.5 & $0.07<$ & $<20$ & 7 & $<0.5$ & $\mathrm{~N}$ & $\mathrm{~N}$ & $\mathrm{~N}$ & $\mathrm{~N}$ & $\mathrm{~N}$ & $<100$ & $\mathrm{~N}$ & $<50$ & $\mathrm{~N}$ & 10 & $\mathrm{~N}$ & 500 & $\mathrm{~N}$ & $\mathrm{~N}$ & $\mathrm{~N}$ & $\mathrm{~N}$ & $\begin{array}{l}\text { Sugary dolomite uniform } \\
\text { crystals with same pyrite }\end{array}$ \\
\hline \multicolumn{2}{|c|}{$\begin{array}{l}\text { Below detection } \\
\text { limits }(\mathrm{N})\end{array}$} & 1 & & 0.3 & 0.002 & 0.001 & 0.025 & 50.01 & 10.005 & 5 & 0.1 & 2 & - & 3 & - & 2 & 2 & 2 & 5 & 10 & 50 & 5 & - & 5 & 5 & \\
\hline \multicolumn{2}{|c|}{$\begin{array}{l}\text { Lower limit of } \\
\text { analysis }\end{array}$} & 2 & & 0.3 & 0.05 & 0.002 & 0.05 & 0.02 & 0.001 & 10 & 0.5 & 5 & 200 & 10 & 20 & 5 & 5 & 5 & 10 & 20 & 100 & 10 & 100 & 10 & 10 & \\
\hline
\end{tabular}


TABLE 2

Semiquantitative Spectrographic Analyses of Samples from Site 226 (Atlantis II Deep)

\begin{tabular}{|c|c|c|c|c|c|c|c|c|c|c|c|c|c|c|c|c|c|c|c|c|c|c|c|c|c|c|c|}
\hline \multirow[b]{2}{*}{ Sample } & \multirow{2}{*}{$\begin{array}{l}\text { Depth } \\
\text { (m) }\end{array}$} & \multicolumn{8}{|c|}{ Major Elements (\%) } & \multicolumn{17}{|c|}{ Minor Elements (ppm) } & \multirow[b]{2}{*}{ Description } \\
\hline & & $\mathrm{Si}$ & $\mathbf{K}$ & Al & $\mathrm{Fe}$ & $\mathrm{Mg}$ & $\mathrm{Ca}$ & $\mathrm{Ti}$ & $\mathrm{Mn}$ & $\mathrm{Ag}$ & As & B & $\mathrm{Ba}$ & $\mathrm{Cd}$ & Co & $\mathrm{Cr}$ & $\mathrm{Cu}$ & Mo & $\mathrm{Ni}$ & $\mathrm{Pb}$ & Sn & $\mathrm{Sr}$ & V & W & $\mathrm{Zn}$ & $\mathrm{Zr}$ & \\
\hline $1-1$ & 1st & 12 & & 3 & $>20$ & 0.15 & 15 & 0.015 & 0.7 & 70 & $\mathrm{~N}$ & 20 & 2000 & 70 & 200 & 10 & 7000 & 100 & 20 & 3000 & $\mathrm{~N}$ & 500 & $70<$ & $50>1$ & 10000 & 10 & $\begin{array}{l}\text { Squeezed bk cake from } \\
\text { red salt material }\end{array}$ \\
\hline $1-1$ & 1st & $\mathrm{N}$ & & 0.3 & $\mathrm{~N}$ & 0.3 & 2 & $\mathrm{~N}$ & 0.01 & $\mathrm{~N}$ & $\mathrm{~N}$ & $\mathrm{~N}$ & $\mathrm{~N}$ & $\mathrm{~N}$ & $\mathrm{~N}$ & $\mathrm{~N}$ & $\mathrm{~N}$ & $\mathrm{~N}$ & $\mathrm{~N}$ & $\mathrm{~N}$ & $\mathrm{~N}$ & 150 & $\mathrm{~N}$ & $\mathrm{~N}$ & $\mathrm{~N}$ & $\mathrm{~N}$ & Salt residue \\
\hline $1-2,15-17$ & 1.6 & 12 & & 5 & 10 & 2 & 5 & 0.7 & 0.2 & 50 & $\mathrm{~N}$ & $\mathrm{~N}$ & 1000 & 50 & 100 & 200 & 5000 & 10 & 100 & 2000 & $\mathrm{~N}$ & 200 & $200 \leq$ & 50 & 1000 & 15 & Dk anhydrite-goethite \\
\hline $1-2,110$ & 26 & 10 & & 2 & 15 & 1.5 & 5 & 0.005 & 0.1 & 50 & $\mathrm{~N}$ & $\mathrm{~N}$ & 200 & $\mathrm{~N}$ & 50 & 10 & 3000 & 5 & 15 & 3000 & $\mathrm{~N}$ & 200 & $\leq 10 \leq$ & $=50$ & 500 & $\mathrm{~N}$ & $\begin{array}{l}\text { Red salt material - } \\
\text { hematite }\end{array}$ \\
\hline $1-3,20-22$ & 3.2 & 2 & & 1 & 5 & 0.3 & $20 \leq$ & $\leq 0.002$ & 0.015 & 30 & $\mathrm{~N} \leq$ & 10 & 5000 & 20 & 30 & $\mathrm{~N}$ & 5000 & 30 & 5 & 300 & $\mathrm{~N}$ & 1000 & 10 & $\mathrm{~N}$ & 500 & $\mathrm{~N}$ & Anhydrite layer-mixed \\
\hline $1-3,30-32$ & 3.3 & 7 & & 2.5 & 15 & 1 & 5 & 0.005 & 0.02 & 100 & $\mathrm{~N}$ & 10 & 200 & 100 & 70 & $\leq 10$ & 7000 & 70 & 10 & 5000 & $\mathrm{~N}$ & 200 & 10 & N 1 & 10000 & $\mathrm{~N}$ & Mixed-mont-anhydrite \\
\hline $1-3,85-87$ & 3.9 & 7 & & 2.5 & 10 & 2 & 3 & 0.007 & 0.02 & 70 & $\mathrm{~N}$ & 10 & 75000 & 70 & 100 & $\leq 10$ & 7000 & 70 & 15 & 3000 & $\mathrm{~N}$ & 1000 & $15 \leq$ & $\leq 501$ & 10000 & $\mathrm{~N}$ & Hematite layer-mixed \\
\hline $1-4,60-62$ & 5.1 & $\leq 2$ & & 1 & 1.5 & $0.3>$ & $>20<$ & $<0.002$ & 0.007 & 3 & $N \leq$ & $\leqslant 10$ & 150 & $\mathrm{~N}$ & 15 & $\mathrm{~N}$ & 1000 & 50 & N & 70 & $\mathrm{~N}$ & 700 & $\leq 10^{-}$ & $\mathrm{N}$ & 1000 & $\mathrm{~N}$ & Anhydrite layer-mixed \\
\hline $1-4,70-72$ & 5.2 & 7 & & 2.5 & 10 & 0.5 & 10 & 0.005 & 0.02 & 50 & $\mathrm{~N}$ & 10 & 100 & 20 & 70 & $\leq 10$ & 7000 & 50 & 10 & 700 & $\mathrm{~N}$ & 300 & $15 \leq$ & 5501 & 10000 & $\mathrm{~N}$ & Bk-mont-hematite \\
\hline $1-4,100-102$ & 5.5 & 2 & & 2 & 20 & 0.5 & 3 & 0.002 & 0.02 & 30 & $\mathrm{~N}$ & 10 & $\mathrm{~N}$ & 5000 & 70 & $\mathrm{~N}$ & 5000 & 200 & $\leq 5$ & 1000 & $\mathrm{~N}$ & 150 & $10 \leq$ & $\leq 50$ & 1500 & $\mathrm{~N}$ & $\begin{array}{l}\text { Hematite-rich layer- } \\
\text { mixed }\end{array}$ \\
\hline $1-4$ & 6 & 20 & & 5 & 10 & 5 & 10 & 0.7 & 0.3 & $\mathrm{~N}$ & $\mathrm{~N}$ & $\mathrm{~N}$ & $\mathrm{~N}$ & $\mathrm{~N}$ & 70 & 300 & 200 & $\mathrm{~N}$ & 150 & $\mathrm{~N}$ & $\mathrm{~N}$ & 100 & 500 & 50 & $\mathrm{~N}$ & 50 & $\begin{array}{l}\text { Fresh glassy looking } \\
\text { basalt }\end{array}$ \\
\hline $1-4$ & 6 & 25 & & $>5$ & 101 & 10 & 10 & 0.7 & 0.3 & 2 & $\mathrm{~N}$ & $\mathrm{~N}$ & 20 & $\mathrm{~N}$ & 70 & 500 & 200 & $\mathrm{~N}$ & 200 & $\mathrm{~N}$ & $\mathrm{~N}$ & 100 & 500 & $\mathrm{~N}$ & $\mathrm{~N}$ & 30 & Altered-looking basalt \\
\hline $1, \mathrm{CC}$ & 6 & 2 & & 1.5 & 0.3 & 1 & 20 & 0.002 & 0.02 & $\mathrm{~N}$ & $\mathrm{~N}$ & $\mathrm{~N}$ & 2000 & $\mathrm{~N}$ & $\mathrm{~N}$ & $\mathrm{~N}$ & 700 & 50 & $\mathrm{~N}$ & 70 & $\mathrm{~N}$ & 1000 & $<10$ & $\mathrm{~N}$ & $\mathrm{~N}$ & $\mathrm{~N}$ & Anhydrite crystals \\
\hline
\end{tabular}

Note:

See Table 1 for explanation of abbreviations.

Sample 1-1 refers to sample squeezed for pore fluid analysis, removing more than $3 / 4$ of contained brine. Other samples contain dried pore fluid whose salts may greatly dilute sediment. 
TABLE $3 \mathrm{~A}$

Semiquantitative Spectrographic Analyses of Samples from Site 227

\begin{tabular}{|c|c|c|c|c|c|c|c|c|c|c|c|c|c|c|c|c|c|c|c|c|c|c|c|c|c|c|}
\hline \multirow[b]{2}{*}{ Sample } & \multirow{2}{*}{$\begin{array}{c}\text { Depth } \\
\text { (M) }\end{array}$} & \multicolumn{8}{|c|}{ Major Elements (\%) } & \multicolumn{16}{|c|}{ Minor Elements (ppm) } & \multirow[b]{2}{*}{ Description } \\
\hline & & $\mathrm{Si}$ & K & Al & $\mathrm{Fe}$ & $\mathrm{Mg}$ & $\mathrm{Ca}$ & $\mathrm{Ti}$ & Mn & $\mathrm{Ag}$ & As & B & $\mathrm{Ba}$ & Cd & Co & $\mathrm{Cr}$ & $\mathrm{Cu}$ & Mo & $\mathrm{Ni}$ & $\mathrm{Pb}$ & $\mathrm{Sn}$ & $\mathrm{Sr}$ & V & $\mathrm{Zn}$ & $\mathrm{Zr}_{\mathrm{r}}$ & \\
\hline $1, \mathrm{CC}$ & 3 & $\mathrm{~N}$ & & $\mathrm{~N}$ & $\mathrm{~N}$ & $<0.02$ & 7 & $<0.002$ & $\mathrm{~N}$ & $\mathrm{~N}$ & $\mathrm{~N}$ & $\mathrm{~N}$ & $\mathrm{~N}$ & $\mathrm{~N}$ & $\mathrm{~N}$ & $\mathrm{~N}$ & $\mathrm{~N}$ & $\mathrm{~N}$ & $\mathrm{~N}$ & $\mathrm{~N}$ & $\mathrm{~N}$ & 3000 & 10 & & $\mathrm{~N}$ & $\begin{array}{l}\text { Radiaxial fibrous } \\
\text { "calcite" }\end{array}$ \\
\hline $1, \mathrm{CC}$ & 3 & 3 & & 2.5 & 0.7 & 10 & $<20$ & 0.03 & 0.15 & $\mathrm{~N}$ & $N \leq$ & $\leq 10$ & $\mathrm{~N}$ & $\mathrm{~N}$ & 5 & 15 & 50 & $\mathrm{~N}$ & 15 & $\leq 10$ & $\mathrm{~N}$ & 1000 & 15 & $\mathrm{~N}$ & 10 & Mixed lithified carb \\
\hline $2-1,24$ & 18.4 & 5 & & $2>$ & $>\mathbf{2 0}$ & 0.02 & 2 & 0.015 & 0.005 & $\mathrm{~N}$ & $\leq 30$ & 50 & $\mathrm{~N}$ & $\mathrm{~N}$ & 5 & 7 & 305 & 500 & 20 & $\mathrm{~N}$ & $\mathrm{~N}$ & 1.0 & 20 & $\mathrm{~N}$ & 10 & Pyrite "brassy" \\
\hline $2-1,140$ & 19.4 & 15 & & 5 & 5 & 5 & 20 & 0.5 & 0.5 & $\mathrm{~N}$ & $\leq 10$ & 20 & 100 & $\mathrm{~N}$ & 20 & 100 & 50 & $\mathrm{~N}$ & 100 & 10 & $\mathrm{~N}$ & 1000 & 30 & $\mathrm{~N}$ & 50 & Lt gy carb mud \\
\hline $3-1,30$ & 27.3 & 15 & & 5 & 2 & 7 & 20 & 0.3 & 0.3 & $\mathrm{~N}$ & $\leq 10$ & 20 & 100 & $\mathrm{~N}$ & 10 & 100 & 70 & $\mathrm{~N}$ & 50 & $\leq 10$ & $\mathrm{~N}$ & 1000 & 30 & $\mathrm{~N}$ & 50 & Bk gy carb mud \\
\hline $3-1,120$ & 28.2 & 15 & & 5 & 2 & 5 & 20 & 0.3 & 0.3 & $\mathrm{~N}$ & $\mathrm{~N}$ & 15 & 10 & $\mathrm{~N}$ & 10 & 100 & 50 & $\mathrm{~N}$ & 70 & 10 & $\mathrm{~N}$ & 1000 & 30 & $\mathrm{~N}$ & 30 & Olive gy carb mud \\
\hline $3-1,145$ & 28.4 & 15 & 0.5 & 5 & 5 & 5 & 20 & 0.5 & 0.5 & $\mathrm{~N}$ & $\leq 10$ & 20 & 200 & $\mathrm{~N}$ & 200 & 100 & 700 & 30 & 300 & 15 & $\mathrm{~N}$ & 1000 & 500\{ & $\left\{\begin{array}{l}400 * \\
\leq 200\end{array}\right.$ & * 50 & Bk gy mud \\
\hline $3-1,148$ & 28.5 & 15 & & $>5$ & 5 & 7 & 20 & 0.5 & 0.5 & $\mathrm{~N}$ & $N \leq$ & $\leq 10$ & 100 & $\mathrm{~N}$ & 20 & 100 & 30 & $\mathrm{~N}$ & 70 & 10 & $\mathrm{~N}$ & 70 & 20 & $\mathrm{~N}$ & 50 & Lt gy carb mud \\
\hline 3, CC1 & 36 & 20 & & 3 & $>20$ & 0.05 & 1.5 & 0.15 & 0.2 & $\mathrm{~N}$ & 500 & 20 & 100 & $\mathrm{~N}$ & 100 & 30 & 300 & 150 & 3007 & 7000 & $\mathrm{~N}$ & $\mathrm{~N}$ & 20 & 700 & $\mathrm{~N}$ & $\begin{array}{l}\mathrm{HC} 1 \text { treatment-no } \\
\text { bromoform }\end{array}$ \\
\hline $3, \mathrm{CC}$ & 36 & 25 & & $>5$ & 15 & 1.5 & 2 & 0.5 & 0.07 & $\mathrm{~N}$ & $\mathrm{~N}$ & 10 & 300 & $\mathrm{~N}$ & 50 & 100 & 150 & 7 & 150 & $\leq 10$ & $\mathrm{~N}$ & 150 & 30 & $\leq 200$ & 70 & $\begin{array}{l}\text { Bromoform separation } \\
+\mathrm{HC} 1 \text { (lights); also } \\
\leq 5 \mathrm{ppm} \mathrm{W}\end{array}$ \\
\hline $3, \mathrm{CC}$ & 36 & 7 & & 1.5 & $>20$ & $\leq 0.02$ & 0.7 & 0.15 & 0.1 & $\mathrm{~N} \quad 10$ & 1000 & 10 & $\mathrm{~N}$ & $\mathrm{~N}$ & 150 & 50 & 200 & 50 & 200 & $\leq 10$ & 10 & $\mathrm{~N}$ & 10 & 200 & 100 & $\begin{array}{l}\text { Bromoform separation } \\
\text { plus HC1 (heavies) }\end{array}$ \\
\hline $6-1,80-81$ & 45.8 & 15 & & $>5$ & 5 & 5 & 20 & 0.5 & 0.5 & $\mathrm{~N}$ & $\mathrm{~N}$ & 10 & 100 & $\mathrm{~N}$ & 20 & 100 & 30 & $\mathrm{~N}$ & 70 & $\leq 10$ & $\mathrm{~N}$ & 100 & 30 & $\mathrm{~N}$ & 70 & Olive grn carb mud \\
\hline $6-2,112-113$ & 347.6 & 12 & & 5 & 5 & 2 & 20 & 0.3 & 0.2 & $\mathrm{~N}$ & $\mathrm{~N}$ & 10 & 130 & $\mathrm{~N}$ & 50 & 70 & 100 & 20 & 150 & 20 & $\mathrm{~N}$ & 1500 & 50 & $\mathrm{~N}$ & 20 & Lt gy carb mud \\
\hline $11, \mathrm{CC}$ & 81 & 7 & & 1 & $>20$ & 0.02 & 1 & 0.005 & 0.015 & $\mathrm{~N}$ & 200 & 30 & $\mathrm{~N}$ & $\mathrm{~N}$ & 70 & $\mathrm{~N}$ & 200 & 30 & 300 & $\mathrm{~N}$ & $\mathrm{~N}$ & $\mathrm{~N}$ & 10 & $500 \leq$ & $\leq 20$ & $\begin{array}{l}\text { Pyrite conglomerate } \\
\text { (wh) }\end{array}$ \\
\hline $12-2,0-10$ & 82.6 & 20 & & $>5$ & 7 & 2 & 15 & 0.5 & 0.2 & $\mathrm{~N}$ & $<10$ & 15 & 200 & $\mathrm{~N}$ & 20 & 150 & 100 & 5 & 100 & $\mathrm{~N}$ & $\mathrm{~N}$ & 700 & 70 & $\leq 200$ & 50 & $\begin{array}{l}\text { Squeezed cake from } \\
\text { interstitial water } \\
\text { sample }\end{array}$ \\
\hline $12-2,20-21$ & 82.7 & 20 & & $>5$ & 5 & 2 & 10 & 0.7 & 0.15 & $\mathrm{~N}$ & $\mathrm{~N}$ & 20 & 200 & $\mathrm{~N}$ & 20 & 200 & 100 & $\mathrm{~N}$ & 150 & $\mathrm{~N}$ & $\mathrm{~N}$ & 300 & 100 & $\mathrm{~N}$ & 70 & Gy carb mud \\
\hline $12-2,77$ & 83.3 & 12 & & 5 & 5 & 1.5 & 20 & 0.3 & 0.15 & $0.5^{*}$ & $\mathrm{~N}$ & 20 & 50 & $\mathrm{~N}$ & 20 & 70 & 705 & 500 & 100 & 10 & $\mathrm{~N}$ & 1000 & 300 & 45 & 50 & $\mathrm{Bk}$ mud \\
\hline $12-2,100-101$ & 183.5 & 20 & & $>5$ & 5 & 2 & 15 & 0.5 & 0.15 & $\mathrm{~N}$ & $\mathrm{~N}$ & 20 & 150 & $\mathrm{~N}$ & 10 & 200 & 50 & $\mathrm{~N}$ & $100=$ & $\leq 10$ & $\mathrm{~N}$ & 1000 & 30 & $\mathrm{~N}$ & 70 & Gy carb mud \\
\hline $13-1,24-26$ & 90.2 & 12 & & 5 & 3 & 1.5 & 15 & 0.3 & 0.5 & $N \leq$ & $\leq 10$ & 15 & 500 & $\mathrm{~N}$ & 15 & 100 & 50 & 5 & 70 & $\mathrm{~N}$ & $\mathrm{~N}$ & 1500 & 70 & $\mathrm{~N}$ & 50 & Olive gy carb chalk \\
\hline $13-2,32$ & 91.8 & 15 & $0.7 *$ & $>5$ & 5 & 1.5 & 20 & 0.3 & 0.15 & $0.5^{*}$ & $\mathrm{~N}$ & 20 & 500 & $\mathrm{~N}$ & 20 & 100 & 100 & 50 & $100 \leq$ & $\leq 10$ & $\mathrm{~N}$ & 1000 & 200 & 70 & 50 & Dk gy carb mud \\
\hline $13-2,50-52$ & 92 & 15 & & 5 & 5 & 2 & 15 & 0.5 & 0.1 & N & $\mathrm{N}$ & 30 & 150 & $N$ & 20 & 200 & 100 & $\mathrm{~N}$ & $150 \leq$ & $\leq 10$ & $\mathrm{~N}$ & 500 & 150 & $\mathrm{~N}$ & 70 & Lt gy carb mud \\
\hline $13, \mathrm{CC}$ & 99 & 25 & & $>5$ & 5 & 0.1 & 0.5 & 0.15 & 0.007 & $\mathrm{~N}$ & $\mathrm{~N}$ & 10 & 20 & 20 & 20 & 10 & 150 & 5 & 70 & 10 & $\mathrm{~N}$ & 300 & 15 & 1500 & 10 & $\begin{array}{l}\text { Bromoform separation } \\
+ \text { HC1 (lights); also } \\
\leq 50 \mathrm{ppm} \mathrm{W}\end{array}$ \\
\hline $13, \mathrm{CC}$ & 99 & 12 & & 1.5 & 20 & $\leq 0.02$ & $\leq 0.05$ & 50.02 & 0.2 & 1.5 & 500 & 20 & $\mathrm{~N}$ & 50 & 150 & $\mathrm{~N}$ & 700 & 50 & 300 & 15 & $\mathrm{~N}$ & $\leq 100$ & $\leq 10>1$ & 10000 & $\mathrm{~N}$ & $\begin{array}{l}\text { Bromoform separation } \\
+\mathrm{HC} 1 \text { (heavies) }\end{array}$ \\
\hline $13, \mathrm{CC}$ & 99 & 20 & & 5 & 5 & 1.5 & 1 & 0.5 & 0.01 & $\mathrm{~N}$ & $\mathrm{~N}$ & 50 & 150 & $<500$ & 10 & 100 & 500 & $\mathrm{~N}$ & 100 & 10 & $\mathrm{~N}$ & 100 & $50>1$ & 10000 & 50 & $\begin{array}{l}\text { HC1 treatment-no } \\
\text { bromoform }\end{array}$ \\
\hline $15, \mathrm{CC}$ & 113 & $\leq 10$ & & $\leq 10.5$ & 20 & $\mathrm{~N}$ & 1 & 0.005 & 0.002 & $\leq 1$ & 2000 & 20 & $\mathrm{~N}$ & $\mathrm{~N}$ & 100 & $\mathrm{~N}$ & 70 & 70 & 300 & $\mathrm{~N}$ & $\mathrm{~N}$ & $\mathrm{~N}$ & 20 & 2000 & $\mathrm{~N}$ & $\begin{array}{l}\text { Pyrite conglomerate } \\
\text { (wh) }\end{array}$ \\
\hline $15-1,93-94$ & 108.9 & 15 & & 5 & 7 & 2 & 15 & 0.3 & 0.2 & $\mathrm{~N}$ & $\mathrm{~N}$ & 50 & 100 & $\mathrm{~N}$ & 50 & 150 & 100 & $\mathrm{~N}$ & $100 \leq$ & $\leq 10$ & $\mathrm{~N}$ & 1500 & 50 & $\mathrm{~N}$ & 50 & $\begin{array}{l}\text { Dk olive grn carb } \\
\text { mud }\end{array}$ \\
\hline $16-2,20-21$ & 114.7 & 15 & & 5 & 5 & 2 & 20 & 0.3 & 0.3 & $\mathrm{~N}$ & $\mathrm{~N}$ & 30 & 500 & $\mathrm{~N}$ & 50 & 100 & 50 & $\mathrm{~N}$ & $100 \leq$ & $\leq 10$ & $\mathrm{~N}$ & 1000 & 100 & $\mathrm{~N}$ & 50 & Dk gy mud \\
\hline $16-2,98-99$ & 115.5 & 15 & & 5 & 5 & 3 & 20 & 0.3 & 0.5 & $\mathrm{~N}$ & $\mathrm{~N}$ & 15 & 50 & $\mathrm{~N}$ & 15 & 100 & 20 & $\mathrm{~N}$ & $70 \leq$ & $\leq 10$ & $\mathrm{~N}$ & 700 & 30 & $\mathrm{~N}$ & 50 & Lt gy carb mud \\
\hline $16-2,110$ & 115.6 & 15 & & 5 & 3 & 2 & 20 & 0.5 & 0.5 & $\mathrm{~N}$ & $\mathrm{~N}$ & 15 & 100 & $\mathrm{~N}$ & 15 & 100 & 50 & $\mathrm{~N}$ & 70 & 10 & $\mathrm{~N}$ & 500 & 30 & $\mathrm{~N}$ & 7 & Olive gy carb chalk \\
\hline $17-1,85$ & 122.8 & 12 & & $>5$ & 2 & 1.5 & $>20$ & 0.2 & 0.2 & $\mathrm{n} \mathrm{N}$ & 10 & 10 & 300 & $\mathrm{~N}$ & 20 & 100 & 50 & $\mathrm{~N}$ & $70 \leq$ & $\leq 10$ & $\mathrm{~N}$ & 1500 & 150 & 200 & 20 & Dk carb layer \\
\hline $18-2,122$ & 133.7 & 15 & & $>5$ & 3 & 2 & 10 & 0.5 & 0.15 & $<0.5^{*}<$ & $<10$ & 20 & 150 & $\mathrm{~N}$ & 20 & 150 & 100 & $\mathrm{~N}$ & $150 \leq$ & $\leq 10$ & $\mathrm{~N}$ & 700 & 300 & $\leqslant 200$ & * 70 & $\begin{array}{l}\text { Dk gy carb mud; also } \\
\leq 50 \mathrm{ppm} \mathrm{W}\end{array}$ \\
\hline
\end{tabular}




\begin{tabular}{|c|c|c|c|c|c|c|c|c|c|c|c|c|c|c|c|c|c|c|c|c|c|c|c|c|c|c|}
\hline \multirow[b]{2}{*}{ Sample } & \multirow{2}{*}{$\begin{array}{c}\text { Depth } \\
(\mathrm{m})\end{array}$} & \multicolumn{8}{|c|}{ Major Elements (\%) } & \multicolumn{16}{|c|}{ Minor Elements (ppm) } & \multirow[b]{2}{*}{ Description } \\
\hline & & $\mathrm{Si}$ & $\mathrm{K}$ & $\mathrm{Al}$ & $\mathrm{Fe}$ & $\mathrm{Mg}$ & $\mathrm{Ca}$ & $\mathrm{Ti}$ & $\mathrm{Mn}$ & $\mathrm{Ag}$ & As & B & $\mathrm{Ba}$ & $\mathrm{Cd}$ & Co & $\mathrm{Cr}$ & $\mathrm{Cu}$ & Mo & $\mathrm{Ni}$ & $\mathrm{Pb}$ & $\mathrm{Sn}$ & Sr & $\mathrm{V}$ & $\mathrm{Zn}$ & $\mathrm{Zr}$ & \\
\hline $18-3,130$ & 135.8 & 12 & $>5$ & & 2 & 1.5 & 20 & 0.2 & 0.15 & $0.5 *$ & * $\mathrm{N}$ & 15 & 70 & $\mathrm{~N}$ & 10 & 100 & 70 & 20 & 100 & $\leq 10$ & $\mathrm{~N}$ & 1500 & 500 & $75^{*}$ & 20 & Bk carb mud \\
\hline $19-2,15$ & 141.7 & 15 & $>5$ & & 5 & 1.5 & 15 & 0.5 & 0.15 & $\mathrm{~N}$ & $\mathrm{~N}$ & 20 & 150 & $\mathrm{~N}$ & 15 & 150 & 70 & $\mathrm{~N}$ & 100 & $\leq 10$ & $\mathrm{~N}$ & 1000 & 70 & $\mathrm{~N}$ & 50 & Lt gy carb mud \\
\hline $20-2,82$ & 151.3 & 12 & $>5$ & & 2 & 1.5 & 15 & 0.3 & 0.15 & $\mathrm{~N}$ & $\mathrm{~N}$ & 20 & 200 & $\mathrm{~N}$ & 15 & 70 & 70 & 7 & 70 & $\leq 10$ & $\mathrm{~N}$ & 1500 & 100 & $\mathrm{~N}$ & 20 & Dk carb chalk \\
\hline $20-3,77$ & 152.8 & 15 & $>5$ & & 10 & 1.5 & 15 & 0.3 & 0.15 & $\mathrm{~N}$ & $\mathrm{~N}$ & 30 & 100 & $\mathrm{~N}$ & 50 & 150 & 100 & 50 & 200 & 10 & $\mathrm{~N}$ & 1000 & 200 & $\mathrm{~N}$ & 30 & $\begin{array}{l}\text { Gy dk chalk? also } \\
\leq 50 \mathrm{ppm} \mathrm{W}\end{array}$ \\
\hline $20-3,77$ & 152.8 & 15 & $>5$ & & 10 & 1 & 10 & 0.3 & 0.1 & $0.5^{*}$ & $\mathrm{~N}$ & 20 & 70 & $\mathrm{~N}$ & 50 & 100 & 100 & 30 & 15 & 15 & $\mathrm{~N}$ & 700 & $100 \leqslant$ & $\leqslant 200^{*}$ & 20 & $\begin{array}{l}\text { Dk carb chalk; also } \\
\leq 50 \text { ppm W }\end{array}$ \\
\hline $20-3,90$ & 152.9 & 15 & $>5$ & & 7 & 1.5 & 15 & 0.3 & 0.1 & $\mathrm{~N}$ & $\mathrm{~N}$ & 20 & 100 & $\mathrm{~N}$ & 20 & 100 & 100 & 70 & 100 & 10 & $\mathrm{~N}$ & 1000 & 500 & $\mathrm{~N}$ & 20 & Dk gy carb chalk \\
\hline $20-3,143$ & 153.4 & 20 & $>5$ & & 5 & 2 & 10 & 0.5 & 0.1 & $\mathrm{~N}$ & $<10$ & 30 & 150 & $\mathrm{~N}$ & 20 & 200 & 100 & $\mathrm{~N}$ & 100 & $\leq 10$ & $\mathrm{~N}$ & 300 & 150 & $\mathrm{~N}$ & 100 & Lt gy carb chalk \\
\hline $20-4,53$ & 154 & 12 & $>5$ & & 15 & 1 & 15 & 0.3 & 0.1 & $\mathrm{~N}$ & $<10$ & 20 & 50 & $\mathrm{~N}$ & 100 & 50 & 150 & 200 & 300 & 50 & $\mathrm{~N}$ & 500 & 150 & $\mathrm{~N}$ & 20 & $\begin{array}{l}\text { Dk gy carb chalk; } \\
\text { also } \leq 50 \mathrm{ppm} \mathrm{W}\end{array}$ \\
\hline $20-5,36$ & 155.4 & 15 & $0.6^{*}>5$ & & 7 & 1.5 & 10 & 0.3 & 0.07 & $0.5^{*}$ & $\mathrm{~N}$ & 30 & 500 & $\mathrm{~N}$ & 20 & 100 & 100 & 200 & 100 & $\leq 10$ & $\mathrm{~N}$ & 500 & 500 & $60 *$ & 30 & Dk gy carb chalk \\
\hline $22-2,100$ & 160.5 & 12 & $>5$ & & 7 & 1.5 & 20 & 0.3 & 0.1 & $\mathrm{~N}$ & $\mathrm{~N}$ & 20 & 100 & $\mathrm{~N}$ & 20 & 100 & 100 & 50 & 100 & $\leq 10$ & $\mathrm{~N}$ & 1000 & 500 & $\mathrm{~N}$ & 20 & Dk gy carb chalk \\
\hline $22-4,40$ & 162.9 & 15 & $>5$ & & 5 & 1.5 & 15 & 0.3 & 0.5 & $\mathrm{~N}$ & $\mathrm{~N}$ & 20 & 500 & $\mathrm{~N}$ & 30 & 100 & 100 & 100 & 100 & $\leq 10$ & $\mathrm{~N}$ & 1500 & 300 & $\mathrm{~N}$ & 30 & Gy chalk \\
\hline $22-4,69$ & 163.2 & 15 & $>5$ & & 7 & 2 & 10 & 0.5 & 0.07 & $\mathrm{~N}$ & $\mathrm{~N}$ & 20 & 150 & $\mathrm{~N}$ & 20 & 200 & 100 & $\mathrm{~N}$ & 150 & $\mathrm{~N}$ & $\mathrm{~N}$ & 500 & 200 & $\mathrm{~N}$ & 70 & Gy chalk \\
\hline $22-4,100$ & 163.5 & 7 & & 2 & 20 & 0.02 & 1 & $0.03 c$ & 0.05 & $\mathrm{~N}$ & $\mathrm{~N}$ & 20 & $\mathrm{~N}$ & $\mathrm{~N}$ & 20 & 10 & 70 & 300 & 150 & 10 & $\mathrm{~N}$ & $\leq 100$ & 20 & $\mathrm{~N}$ & 70 & Pyrite \\
\hline $24-6,50$ & 184 & 12 & & 3 & 3 & 1 & 15 & $0.2 \mathrm{C}$ & 0.3 & $\mathrm{~N}$ & $\mathrm{~N}$ & 15 & 500 & $\mathrm{~N}$ & 15 & 50 & 100 & 20 & 50 & $\leq 10$ & $\mathrm{~N}$ & 700 & 300 & $\mathrm{~N}$ & 30 & Dk chalk \\
\hline $25-2,39$ & 186.9 & 20 & & 5 & 5 & 1 & 10 & $0.3 \quad \mathrm{C}$ & 0.3 & $\mathrm{~N}$ & $\mathrm{~N}$ & 30 & 100 & $\mathrm{~N}$ & 30 & 100 & 150 & 5 & 70 & $\leq 10$ & $\mathrm{~N}$ & 1500 & 50 & $\mathrm{~N}$ & 50 & Dk chalk \\
\hline $25, \mathrm{CC}$ & 194 & 7 & & $1.5>$ & $20 \leq$ & $\leq 0.1$ & $2 \leq$ & 0.01 & 0.3 & $\mathrm{~N}$ & $\mathrm{~N}$ & $\mathrm{~N}$ & $\mathrm{~N}$ & $\mathrm{~N}$ & 7 & $\mathrm{~N}$ & 100 & $\mathrm{~N}$ & 50 & N & $\mathrm{N}$ & $\mathrm{N}$ & 10 & $\mathrm{~N}$ & $\mathrm{~N}$ & $\begin{array}{l}\text { Yw nodular pyrite } \\
\text { agglomerates }\end{array}$ \\
\hline $26-2,114$ & 196.5 & 20 & $>5$ & & 7 & 1.5 & 2 & 0.7 & 0.015 & $\mathrm{~N}$ & $\mathrm{~N}$ & 50 & 150 & $\mathrm{~N}$ & 30 & 150 & 150 & 5 & 100 & $\mathrm{~N}$ & $\mathrm{~N}$ & 150 & 150 & $\mathrm{~N}$ & 50 & $\begin{array}{l}\text { gr gy chalk? also } \\
\leq 50 \mathrm{ppm} \mathrm{W}\end{array}$ \\
\hline $28-2,46$ & 214 & 15 & $0.9 *>5$ & & 5 & 10 & 5 & 0.5 & 0.1 & $\mathrm{~N}$ & $<10$ & 30 & 100 & $\mathrm{~N}$ & 20 & 150 & 100 & $\mathrm{~N}$ & 70 & $\mathrm{~N}$ & $\mathrm{~N}$ & 100 & 20 & $60^{*}$ & 30 & $\begin{array}{l}\text { Dk dolomite claystone } \\
\text { also } \leq 50 \mathrm{ppm} \mathrm{W}\end{array}$ \\
\hline $29-2,70$ & 223.2 & 15 & $>5$ & 5 & 5 & 7 & 10 & 0.3 & 0.3 & $\mathrm{~N}$ & $\mathrm{~N}$ & 30 & 100 & $\mathrm{~N}$ & 15 & 200 & 100 & 30 & 100 & $\leq 10$ & $\mathrm{~N}$ & 300 & 30 & $\mathrm{~N}$ & 30 & $\begin{array}{l}\text { Med gy grn carb mud; } \\
\text { also } \leq 50 \mathrm{ppm} \mathrm{W}\end{array}$ \\
\hline $31-1,110$ & 236.1 & 15 & $>5$ & 5 & 5 & 5 & 10 & 0.3 & 0.3 & $\mathrm{~N}$ & $\mathrm{~N}$ & 500 & 100 & $\mathrm{~N}$ & 20 & 100 & 100 & 5 & 70 & $\leq 10$ & $\mathrm{~N}$ & 1000 & 70 & $\mathrm{~N}$ & 30 & $\begin{array}{l}\text { Olive gy mud; also } \\
\leq 50 \mathrm{ppm} \mathrm{W}\end{array}$ \\
\hline $31-1,120$ & 236.2 & 15 & $1.1 *>5$ & & 5 & 5 & 7 & 0.3 & 0.2 & N & $\mathrm{N}$ & 200 & 100 & $\mathrm{~N}$ & 15 & 100 & 70 & 5 & 70 & $\leq 10$ & $\mathrm{~N}$ & 500 & 50 & 80 & 50 & $\begin{array}{l}\text { Bk shale; also } \\
\leq 50 \text { ppm W }\end{array}$ \\
\hline $31-$, CC & 244 & $\mathrm{~N}$ & & 0.3 & $\mathrm{~N}$ & 5 & 0.3 & $\mathrm{~N}$ & $\mathrm{~N}$ & $\mathrm{~N}$ & $\mathrm{~N}$ & $\mathrm{~N}$ & $\mathrm{~N}$ & $\mathrm{~N}$ & $\mathrm{~N}$ & $\mathrm{~N}$ & $\mathrm{~N}$ & $\mathrm{~N}$ & $\mathrm{~N}$ & $\mathrm{~N}$ & $\mathrm{~N}$ & $\mathrm{~N}$ & $\leq 10$ & $\mathrm{~N}$ & $\mathrm{~N}$ & $\begin{array}{l}\text { Redd ish material in } \\
\text { salt }\end{array}$ \\
\hline $31, \mathrm{CC}$ & 244 & $\mathrm{~N}$ & & 1 & $N>$ & 10 & 2 & $\mathrm{~N}$ & 0.001 & $\mathrm{~N}$ & $\mathrm{~N}$ & $\leq 10$ & $\mathrm{~N}$ & $\mathrm{~N}$ & $\mathrm{~N}$ & $\mathrm{~N}$ & 20 & $\mathrm{~N}$ & $\mathrm{~N}$ & $\mathrm{~N}$ & $\mathrm{~N}$ & 150 & $\leq 10$ & $\mathrm{~N}$ & $\mathrm{~N}$ & Salt \\
\hline $32-2,77$ & 246.3 & 25 & $1.5 * 2$ & 2.5 & 1.5 & 1 & 1 & 0.070 & 0.01 & $\mathrm{~N}$ & $\mathrm{~N}$ & 300 & 20 & $\mathrm{~N}$ & $\leq 5$ & 20 & 100 & 7 & 10 & $\mathrm{~N}$ & $\mathrm{~N}$ & 200 & 50 & $60^{*}$ & 10 & $\begin{array}{l}\text { Dk shale; also } \\
\leq 50 \mathrm{ppm} \mathrm{W}\end{array}$ \\
\hline $32-2,136$ & 246.9 & $\mathrm{~N}$ & & 0.3 & $\mathrm{~N}$ & 0.3 & 11 & $\mathrm{~N}$ & $\mathrm{~N}$ & $\mathrm{~N}$ & $\mathrm{~N}$ & $\mathrm{~N}$ & $\mathrm{~N}$ & $\mathrm{~N}$ & $\mathrm{~N}$ & $\mathrm{~N}$ & $\mathrm{~N}$ & $\mathrm{~N}$ & $\mathrm{~N}$ & $\mathrm{~N}$ & $\mathrm{~N}$ & $\mathrm{~N}$ & $\leq 10$ & $\mathrm{~N}$ & $\mathrm{~N}$ & Salt \\
\hline $32-5,83$ & 250.8 & $\mathrm{~N}$ & & 0.3 & $\mathrm{~N}$ & 0.05 & $0.2 \mathrm{I}$ & $\mathrm{N}$ & $\mathrm{N}$ & $\mathrm{N}$ & $\mathrm{N}$ & $\mathrm{N}$ & $\mathrm{N}$ & $\mathrm{N}$ & $\mathrm{N}$ & $\mathrm{N}$ & $\mathrm{N}$ & $\mathrm{N}$ & $\mathrm{N}$ & $\mathrm{N}$ & $\mathrm{N}$ & $\mathrm{N}$ & $\leq 10$ & $\mathrm{~N}$ & $\mathrm{~N}$ & Salt \\
\hline $33-2,103$ & 255.5 & $\mathrm{~N}$ & & 0.3 & $\mathrm{~N}$ & 0.03 & $0.1 \mathrm{I}$ & $\mathrm{N}$ & $\mathrm{N}$ & $\mathrm{N}$ & $\mathrm{N}$ & $\mathrm{N}$ & $\mathrm{N}$ & $\mathrm{N}$ & $\mathrm{N}$ & $\mathrm{N}$ & $\mathrm{N}$ & $\mathrm{N}$ & $\mathrm{N}$ & $\mathrm{N}$ & $\mathrm{N}$ & $\mathrm{N}$ & $\leq 10$ & $\mathrm{~N}$ & $\mathrm{~N}$ & Salt \\
\hline $36-2,106$ & 282.6 & 30 & $2.4^{*} 5$ & 5 & 7 & 2 & 2 & 0.3 & 0.07 & $\mathrm{~N}$ & $<10$ & 700 & 100 & $\mathrm{~N}$ & 20 & 70 & 200 & 20 & 100 & $\mathrm{~N}$ & $\mathrm{~N}$ & 150 & 150 & $550 *$ & 50 & Bk shale \\
\hline $36-3,3$ & 283 & 30 & & 3 & 2 & 1.5 & 20 & $0.3 \mathrm{C}$ & 0.015 & $\mathrm{~N}$ & $<10$ & 300 & 50 & $\mathrm{~N}$ & 10 & 50 & 150 & 7 & 50 & $\leq 10$ & $\mathrm{~N}$ & 100 & 150 & $\mathrm{~N}$ & 30 & Bk shale \\
\hline $36-3,3-14$ & 285 & 15 & & 0.1 & $\mathrm{~N}$ & 0.05 & $20 \leq$ & $0.002 \mathrm{I}$ & & $\mathrm{N}$ & $\mathrm{N}$ & $\leq 10$ & $\mathrm{~N}$ & $\mathrm{~N}$ & $\mathrm{~N}$ & $\mathrm{~N}$ & 5 & $\mathrm{~N}$ & $\mathrm{~N}$ & $\mathrm{~N}$ & $\mathbf{N}$ & 2000 & 10 & $\mathrm{~N}$ & $\mathrm{~N}$ & $\begin{array}{l}\text { Quartz-bearing } \\
\text { anhydrite }\end{array}$ \\
\hline 37,2 & 290.5 & $\mathrm{~N}$ & $<0.1 *$ & 0.1 & $\mathrm{~N}$ & 1 & 20 & 0.0050 & 0.002 & $\mathrm{~N}$ & $<10$ & 15 & $\mathrm{~N}$ & $\mathrm{~N}$ & $\mathbf{N}$ & $\mathrm{N}$ & $\mathrm{N}$ & $\mathrm{N}$ & $\mathrm{N}$ & $\mathrm{N}$ & $\mathrm{N}$ & 2000 & 10 & $100^{*}$ & $\mathrm{~N}$ & $\begin{array}{l}\text { Anhydrite K-ar } \\
\text { special }\end{array}$ \\
\hline \multirow{3}{*}{$\begin{array}{l}40-2,30 \\
44, C C\end{array}$} & 306.8 & 15 & $<$ & 5 & 7 & 2 & 10 & 0.3 & 0.2 & $\mathrm{~N}$ & $\mathrm{~N}$ & 20 & 150 & $\mathrm{~N}$ & 20 & 150 & 100 & 5 & 70 & 10 & $\mathrm{~N}$ & 500 & 100 & $\mathrm{~N}$ & 30 & \multirow{3}{*}{$\begin{array}{l}\text { Shale gravel } \\
\text { Bk shale; also } \\
\leq 50 \text { ppm W } \\
\text { Salt residue from } \\
\text { interstitial water }\end{array}$} \\
\hline & 350 & 20 & $2.0^{*}<$ & & 10 & 7 & 1 & 0.5 & 0.3 & $\mathrm{~N}$ & $\mathrm{~N}$ & 500 & 150 & $\mathrm{~N}$ & 50 & 100 & 300 & 5 & 100 & $\leq 10$ & $\mathrm{~N}$ & 150 & 1002 & 2000 & 30 & \\
\hline & & $\mathrm{N}$ & & 0.3 & N 1 & 10 & 2 & $\mathbf{N}$ & 0.003 & $\mathrm{~N}$ & $\mathbf{N}$ & 1000 & $\mathrm{~N}$ & $\mathrm{~N}$ & $\mathrm{~N}$ & $\mathrm{~N}$ & $\mathrm{~N}$ & $\mathrm{~N}$ & $\mathrm{~N}$ & $\mathrm{~N}$ & $\mathrm{~N}$ & 700 & $\leq 10$ & $\mathrm{~N}$ & $\mathrm{~N}$ & \\
\hline
\end{tabular}


TABLE 3B

Analysis of Separated Heavy Minerals (from Table 3A)

\begin{tabular}{|c|c|c|c|c|c|c|c|c|c|c|c|c|c|c|c|}
\hline Core & $\mathrm{Sec}$ & $\begin{array}{l}\text { Interval } \\
(\mathrm{cm})\end{array}$ & $\begin{array}{l}\text { Depth } \\
\text { (m) }\end{array}$ & $\begin{array}{l}\mathrm{Fe} \\
(\%)\end{array}$ & $\begin{array}{r}\mathrm{Mn} \\
(\%)\end{array}$ & $\mathrm{Cu}$ & $\mathrm{Ag}$ & $\mathrm{Pb}$ & $\mathrm{Zn}$ & $\mathrm{Cd}$ & As & Mo & $\mathrm{Ni}$ & Co & Description \\
\hline 2 & & $\mathrm{CC}$ & 3 & $>20$ & 0.005 & 30 & $\mathrm{~N}$ & $\mathrm{~N}$ & $\mathrm{~N}$ & $\mathrm{~N}$ & $\mathrm{~N}$ & 500 & 20 & 5 & Brassy pyrite \\
\hline 3 & & $\mathrm{CC}$ & 36 & $>20$ & 0.2 & 300 & $\mathrm{~N}$ & 7000 & 700 & $\mathrm{~N}$ & 500 & 150 & 300 & 100 & $\begin{array}{l}\text { Residue after HC1 treatment } \\
\text { (chiefly framboidal pyrite) }\end{array}$ \\
\hline 3 & & $\mathrm{CC}$ & 36 & $>20$ & 0.1 & 200 & $\mathrm{~N}$ & $\leq 10$ & 200 & $\mathrm{~N}$ & 1000 & 50 & 200 & 150 & $\begin{array}{l}\text { Bromoform and } \mathrm{HC} 1 \text { separation } \\
\text { mainly pyrite. Also } 10 \mathrm{ppm} \mathrm{Sn}\end{array}$ \\
\hline 11 & & $\mathrm{CC}$ & 81 & $>20$ & 0.015 & 200 & $\mathrm{~N}$ & $\mathrm{~N}$ & 500 & $\mathrm{~N}$ & 200 & 30 & 300 & 70 & $\begin{array}{l}\text { Silver white pyrite aggregate } \\
\text { (layer) }\end{array}$ \\
\hline 13 & & $\mathrm{CC}$ & 99 & 5 & 0.01 & 500 & $\mathrm{~N}$ & 10 & $>1 \%$ & $>500$ & $\mathrm{~N}$ & $\mathrm{~N}$ & 100 & 10 & Sphalerite (HC1 residue) \\
\hline 13 & & CC & 99 & $>20$ & 0.2 & 700 & 1.5 & 15 & $>1 \%$ & 50 & 500 & 50 & 300 & 150 & $\begin{array}{l}\text { Sphalerite (bromoform \& } \mathrm{HC} 1 \text { ) } \\
\text { and pyrite }\end{array}$ \\
\hline 15 & & $\mathrm{CC}$ & 113 & $>20$ & 0.002 & 70 & $\leq 1$ & $\mathrm{~N}$ & 2000 & $\mathrm{~N}$ & 2000 & 70 & 300 & 100 & Silver white pyrite \\
\hline 22 & 4 & & 163 & $>20$ & 0.005 & 300 & $\mathrm{~N}$ & 10 & $\mathrm{~N}$ & $\mathrm{~N}$ & $\mathrm{~N}$ & 300 & 150 & 20 & Pyrite, yellowish \\
\hline 25 & & $\mathrm{CC}$ & 194 & $>20$ & 0.3 & 100 & $\mathrm{~N}$ & $\mathrm{~N}$ & $\mathrm{~N}$ & $\mathrm{~N}$ & $\mathrm{~N}$ & $\mathrm{~N}$ & 50 & 7 & $\begin{array}{l}\text { Yellow nodular pyrite } \\
\text { agglomerates }\end{array}$ \\
\hline
\end{tabular}


TABLE 4

Semiquantitative Spectrographic Analyses of Samples from Site 228

\begin{tabular}{|c|c|c|c|c|c|c|c|c|c|c|c|c|c|c|c|c|c|c|c|c|c|c|c|c|c|c|}
\hline \multirow[b]{2}{*}{ Sample } & \multirow{2}{*}{$\begin{array}{c}\text { Depth } \\
(\mathrm{M}) \\
\end{array}$} & \multicolumn{8}{|c|}{ Major Elements (\%) } & \multicolumn{16}{|c|}{ Minor Elements (ppm) } & \multirow[b]{2}{*}{ Description } \\
\hline & & $\mathrm{Si}$ & $\mathbf{K}$ & $\mathrm{Al}$ & $\mathrm{Fe}$ & $\mathrm{Mg}$ & $\mathrm{Ca}$ & $\mathrm{Ti}$ & $\mathrm{Mn}$ & $\mathrm{Ag}$ & As & B & $\mathrm{Ba}$ & $\mathrm{Cd}$ & Co & $\mathrm{Cr}$ & $\mathrm{Cu}$ & Mo & $\mathrm{Ni}$ & $\mathrm{Pb}$ & Sn & Sr & V & $\mathrm{Zn}$ & $\mathrm{Zr}$ & \\
\hline $1-1,96$ & 1 & 15 & & 5 & 7 & 3 & 10 & 0.5 & 0.2 & $\mathrm{~N}$ & $\leq 10$ & 15 & 200 & $\mathrm{~N}$ & 20 & 100 & 100 & $\mathrm{~N}$ & 70 & 10 & $\mathrm{~N}$ & 700 & 100 & $\mathrm{~N}$ & 100 & Gy carb ooze \\
\hline $1-2,46$ & 2 & 25 & & $>5$ & 10 & 5 & 5 & 0.7 & 0.15 & $\mathrm{~N}$ & $\mathrm{~N}$ & 20 & 500 & $\mathrm{~N}$ & 20 & 200 & 100 & $\mathrm{~N}$ & 100 & 10 & $\mathrm{~N}$ & 500 & 150 & $\mathrm{~N}$ & 200 & Dk gy carb ooze \\
\hline $1-3,51$ & 3.5 & 15 & & 5 & 5 & 5 & 20 & 0.3 & 0.2 & $\mathrm{~N}$ & $\mathrm{~N}$ & 15 & 150 & $\mathrm{~N}$ & 15 & 100 & 50 & $\mathrm{~N}$ & 70 & 10 & $\mathrm{~N}$ & 1000 & 70 & N 1 & 100 & Lt gy carb ooze \\
\hline $1-3,86$ & 3.9 & 15 & & 5 & 5 & 3 & 20 & 0.3 & 0.2 & $\mathrm{~N}$ & $\mathrm{~N}$ & 20 & 150 & $\mathrm{~N}$ & 20 & 150 & 50 & $\mathrm{~N}$ & 100 & 10 & $\mathrm{~N}$ & 1000 & 50 & $\mathrm{~N}$ & 70 & Lt gy carb ooze \\
\hline $1-5,81-82$ & 6.8 & 15 & & 5 & 7 & 7 & 20 & 0.5 & 0.5 & $\mathrm{~N}$ & $\mathrm{~N}$ & 20 & 150 & $\mathrm{~N}$ & 15 & 150 & 50 & $\mathrm{~N}$ & 100 & 10 & $\mathrm{~N}$ & 1000 & 50 & $\mathrm{~N}$ & 100 & Lt brn carb ooze \\
\hline $1, \mathrm{CC}$ & 5 & 30 & & 5 & 5 & 3 & 2 & 0.5 & 0.07 & $\mathrm{~N}$ & $\mathrm{~N}$ & N & 700 & $\mathrm{~N}$ & 15 & 100 & 100 & $\mathrm{~N}$ & 70 & 15 & $\mathrm{~N}$ & 300 & 70 & $\mathrm{~N}$ & 100 & $\begin{array}{l}\text { Bromoform + HC1 } \\
\text { separation (lights) }\end{array}$ \\
\hline $1, \mathrm{CC}$ & 5 & 15 & & 5 & 20 & 5 & 5 & 0.7 & 0.3 & $\mathrm{~N}$ & $\mathrm{~N}$ & 10 & 70 & $\mathrm{~N}$ & 50 & 500 & 100 & $\mathrm{~N}$ & 100 & 10 & $\mathrm{~N}$ & 500 & 500 & N 10 & 000 & $\begin{array}{l}\text { Bromoform + } \mathrm{HC} 1 \\
\text { separation (heavies) }\end{array}$ \\
\hline $6-5,93-95$ & 48.9 & 25 & & 5 & 7 & 5 & 20 & 0.7 & 0.15 & $\mathrm{~N}$ & 10 & 20 & 300 & $\mathrm{~N}$ & 30 & 150 & 100 & $\mathrm{~N}$ & 150 & 10 & $\mathrm{~N}$ & 500 & 300 & $\mathrm{~N}$ & 500 & Gy detrital sandy silt \\
\hline $7-6,125-127$ & 59.2 & 20 & & 5 & 7 & 3 & 15 & 0.5 & 0.5 & $\mathrm{~N}$ & $\mathrm{~N}$ & 15 & 200 & $\mathrm{~N}$ & 15 & 150 & 50 & $\mathrm{~N}$ & $70 \leq$ & $\leq 10$ & $\mathrm{~N}$ & 1000 & 150 & $\mathrm{~N}$ & 100 & Lt gy ooze \\
\hline $8-3,140-141$ & 63.9 & 25 & & 5 & 7 & 3 & 10 & 0.5 & 0.2 & $\mathrm{~N}$ & $\mathrm{~N}$ & 20 & 200 & $\mathrm{~N}$ & 20 & 150 & 100 & $\mathrm{~N}$ & 100 & 10 & $\mathrm{~N}$ & 700 & 150 & $\mathrm{~N}$ & 200 & Gy ooze \\
\hline $10-6,85-86$ & 77.4 & 15 & & 5 & 5 & 5 & 15 & 0.5 & 0.5 & $\mathrm{~N}$ & $\leq 10$ & 20 & 100 & $\mathrm{~N}$ & 10 & 150 & 30 & $\mathrm{~N}$ & 70 & $\leq 10$ & $\mathrm{~N}$ & 1000 & 100 & $\mathrm{~N}$ & 70 & Lt gy ooze \\
\hline $11-4,120-121$ & 83.7 & 20 & & $>5$ & 7 & 3 & 10 & 0.5 & 0.2 & $\mathrm{~N}=$ & $\leq 10$ & 20 & 200 & $\mathrm{~N}$ & 15 & 150 & 50 & $\mathrm{~N}$ & $70 \leq$ & $\leq 10$ & $\mathrm{~N}$ & 700 & 100 & $\mathrm{~N}$ & 70 & Lt brn ooze \\
\hline $12-4,56$ & 92.1 & 20 & & 5 & 7 & 3 & 10 & 0.5 & 0.5 & $\mathrm{~N}:$ & $\leq 10$ & 20 & 200 & $\mathrm{~N}$ & 20 & 150 & 70 & $\mathrm{~N}$ & 100 & 10 & $\mathrm{~N}$ & 1000 & 200 & $\mathrm{~N}$ & 150 & Reddish brn carb ooze \\
\hline $12-6,75$ & 95.2 & 20 & & $>5$ & 10 & 3 & 10 & 0.7 & 0.3 & $\mathrm{~N}$ & $\mathrm{~N}$ & 20 & 300 & $\mathrm{~N}$ & 30 & 200 & 100 & $\mathrm{~N}$ & 100 & $\leq 10$ & $\mathrm{~N}$ & 700 & 200 & $\mathrm{~N}$ & 150 & Med gy carb ooze \\
\hline $13-2,10$ & 97.6 & 12 & & 5 & 1.5 & 2 & 0.3 & 30.05 & $\mathrm{~N}$ & $\mathrm{~N}$ & 10 & 50 & $\mathrm{~N}$ & $\mathrm{~N}$ & 70 & 15 & $\mathrm{~N}$ & & 100 & 10 & $\mathrm{~N}$ & 5000 & 30 & $\mathrm{~N}$ & 50 & Lithified carb \\
\hline $13-2,110$ & 98.6 & 20 & & $>5$ & 7 & 5 & 10 & 0.7 & 0.2 & $\mathrm{~N}$ & $\mathrm{~N}$ & 20 & 500 & $\mathrm{~N}$ & 20 & 150 & 70 & $\mathrm{~N}$ & 100 & $\leq 10$ & $\mathrm{~N}$ & 500 & 200 & $\mathrm{~N} 2$ & 200 & $\begin{array}{l}\text { Lt gy carb chalk also; } \\
\leq 50 \mathrm{ppm} \mathrm{V}\end{array}$ \\
\hline $13-2,145$ & 98.9 & 12 & $0.7^{*}$ & 5 & 1 & 20 & 15 & 0.3 & 0.3 & $\mathrm{~N}=$ & $\leq 10$ & 30 & 300 & $\mathrm{~N}$ & 100 & 150 & 70 & 100 & 300 & 15 & $\mathrm{~N}$ & 1500 & 300 & $95^{*}$ & 50 & Bk gy d Bk gy carb chalk \\
\hline $14-1,120-121$ & 106.2 & 15 & & $>5$ & 5 & 5 & 15 & 0.5 & 0.3 & $\mathrm{~N}$ & $\mathrm{~N}$ & 30 & 150 & $\mathrm{~N}$ & 15 & 150 & 50 & $\mathrm{~N}$ & 100 & 10 & $\mathrm{~N}$ & 1000 & 50 & $\mathrm{~N}$ & 70 & Lt gy siltstone \\
\hline $142,44-45$ & 106.9 & 15 & & $>5$ & 5 & 5 & 20 & 0.5 & 0.5 & $\mathrm{~N}$ & $\mathrm{~N}$ & 30 & 150 & $\mathrm{~N}$ & 15 & 150 & 50 & $\mathrm{~N}$ & 100 & 10 & $\mathrm{~N}$ & 700 & 50 & N 1 & 100 & Lt gy carb chalk \\
\hline $14-3,818-19$ & 108.2 & 15 & & 5 & 7 & 2 & 20 & 0.5 & 0.3 & $\mathrm{~N}$ & $\mathrm{~N}$ & 30 & 100 & $\mathrm{~N}$ & 50 & 100 & 50 & 5 & 200 & 15 & $\mathrm{~N}$ & 1000 & 100 & $\mathrm{~N}$ & 70 & Dk gy siltstone \\
\hline 143,100 & 109 & 20 & & 5 & 5 & 2 & 15 & 0.7 & 0.2 & $\mathrm{~N}$ & $\mathrm{~N}$ & 30 & 300 & $\mathrm{~N}$ & 20 & 150 & 50 & $\mathrm{~N}$ & 100 & 10 & $\mathrm{~N}$ & 700 & 100 & $\mathrm{~N}$ & 150 & Gy carb siltstone \\
\hline $14-4,82$ & 110.3 & 15 & & $>5$ & 5 & 7 & 20 & 0.5 & 0.7 & $\mathrm{~N}$ & $<10$ & 30 & 100 & $\mathrm{~N}$ & 15 & 150 & 20 & $\mathrm{~N}$ & 70 & 10 & $\mathrm{~N}$ & 1000 & 50 & $\mathrm{~N}$ & 70 & $\begin{array}{l}\text { Lt reddish gy } \\
\text { carb siltstone }\end{array}$ \\
\hline $15-1,98$ & 115 & 12 & & 5 & 3 & 2 & 20 & 0.2 & 0.1 & $\mathrm{~N}$ & $N \leq$ & $\leqslant 15$ & 100 & $\mathrm{~N}$ & 10 & 70 & 30 & 15 & 50 & 10 & $\mathrm{~N}$ & $>5000$ & 50 & & 50 & Pteropod ooze \\
\hline $15-6,114-115$ & 122.6 & 12 & & $>5$ & 7 & 1.5 & 15 & 0.3 & 0.5 & $\mathrm{~N}$ & $\mathrm{~N}$ & 30 & 300 & $\mathrm{~N}$ & 30 & 100 & 100 & 100 & 200 & 10 & $\mathrm{~N}$ & 1500 & 700 & $\mathrm{~N}$ & 50 & Cloudy carb chalk \\
\hline $15, \mathrm{CC}$ & 123 & 7 & & $1>$ & $>20$ & 0.02 & 1.5 & 50.015 & 50.007 & N 3 & 3000 & 30 & $\mathrm{~N}$ & $\mathrm{~N}$ & 20 & 10 & 50 & 200 & 300 & $\mathrm{~N}$ & $\mathrm{~N}$ & $\leq 100$ & 10 & $\mathrm{~N}$ & 10 & Pyrite \\
\hline $16-1,125-126$ & 124.3 & 15 & & $>5$ & 5 & 0.5 & 20 & 0.3 & 0.5 & $\mathrm{~N}$ & $\mathrm{~N}$ & 20 & 200 & $\mathrm{~N}$ & 15 & 150 & 100 & $\mathrm{~N}$ & 70 & 10 & $\mathrm{~N}$ & 1000 & 20 & $\mathrm{~N}$ & 70 & Lt gy carb chalk \\
\hline $16-3,22-23$ & 126.2 & 25 & & $>5$ & 10 & 3 & 15 & 0.7 & 0.3 & $\mathrm{~N}$ & $\mathrm{~N}$ & 30 & 300 & $\mathrm{~N}$ & 50 & 200 & 70 & $\mathrm{~N}$ & 150 & $\mathrm{~N}$ & $\mathrm{~N}$ & 1000 & 300 & N 2 & 200 & Med gy carb chalk \\
\hline $16-5,73-74$ & 129.7 & 25 & & $>5$ & 15 & 5 & 15 & 0.7 & 0.5 & $\mathrm{~N}$ & $\mathrm{~N}$ & 30 & 300 & $\mathrm{~N}$ & 70 & 200 & 100 & $\mathrm{~N}$ & 150 & $\mathrm{~N}$ & $\mathrm{~N}$ & 1000 & 200 & $\mathrm{~N}$ & 200 & Greenish gy carb chalk \\
\hline $16-5$ & 129 & $\mathrm{~N}$ & & $\leq 0.3$ & 2 & 2 & $\mathrm{~N}$ & $\mathrm{~N}$ & $\mathrm{~N}$ & $\mathrm{~N}$ & 15 & $\mathrm{~N}$ & $\mathrm{~N}$ & $\mathrm{~N}$ & $\mathrm{~N}$ & $\mathrm{~N}$ & $\mathrm{~N}$ & $\mathrm{~N}$ & $\mathrm{~N}$ & $\mathrm{~N}$ & $\mathrm{~N}$ & 700 & 10 & $\mathrm{~N} \mathrm{~N}$ & N & $\begin{array}{l}\text { Salt from interstitial } \\
\text { water sample }\end{array}$ \\
\hline $18-1,132-133$ & 142.3 & 30 & & 5 & 10 & 5 & 10 & 0.7 & 0.5 & $\mathrm{~N}$ & $<10$ & 20 & 500 & $\mathrm{~N}$ & 30 & 200 & 100 & $\mathrm{~N}$ & 100 & $\mathrm{~N}$ & $\mathrm{~N}$ & 700 & 200 & $\mathrm{~N}$ & 200 & Olive carb siltstone \\
\hline $18, \mathrm{CC}$ & 150 & 12 & & 3 & 5 & 3 & 20 & 0.3 & 0.15 & $\mathrm{~N}$ & N 1 & 101 & 50 & $\mathrm{~N}$ & 10 & 100 & 20 & 50 & 30 & 10 & $\mathrm{~N}$ & $>5000$ & 30 & & 30 & Pteropod shell ooze \\
\hline $19-1,75$ & 150.7 & 12 & $0.6^{*}$ & 5 & 5 & 1.5 & 15 & 0.2 & 0.5 & $\mathrm{~N}$ & $<10$ & 30 & 700 & $\mathrm{~N}$ & 50 & 70 & 70 & 150 & 300 & 20 & $\mathrm{~N}$ & 1000 & 700 & $20 *$ & 50 & Bk claystone \\
\hline $19-2,112$ & 152.6 & 25 & & $>5$ & 20 & 1.5 & 7 & 0.5 & 0.5 & $\mathrm{~N}$ & $<10$ & 50 & 700 & $\mathrm{~N}$ & 30 & 150 & 100 & 50 & 100 & 10 & $\mathrm{~N}$ & 500 & 300 & 2002 & 200 & Redd ish brn chalk \\
\hline $19-2$ & 151.5 & $\mathrm{~N}$ & & 25 & $\mathrm{~N}$ & 1.5 & & $\leq 0.002$ & $2 \mathrm{~N}$ & $\mathrm{~N}$ & $\mathrm{~N}$ & $\mathrm{~N}$ & $\mathrm{~N}$ & $\mathrm{~N}$ & $\mathrm{~N}$ & $\mathrm{~N}$ & $\mathrm{~N}$ & $\mathrm{~N}$ & $\mathrm{~N}$ & $\mathrm{~N}$ & $\mathrm{~N}$ & 700 & 10 & $\mathrm{~N}$ & N & $\begin{array}{l}\text { Salt from interstitial } \\
\text { water sample }\end{array}$ \\
\hline $20-4,120-121$ & 160.7 & 15 & & 5 & 7 & 2 & 10 & 0.3 & 0.7 & $\mathrm{~N}$ & $\mathrm{~N}$ & 50 & 150 & $\mathrm{~N}$ & 50 & 100 & 70 & $\mathrm{~N}$ & 150 & 10 & $\mathrm{~N}$ & 1500 & 100 & $\mathrm{~N}$ & 50 & Dk gy carb chalk \\
\hline
\end{tabular}


TABLE 4 - Continued

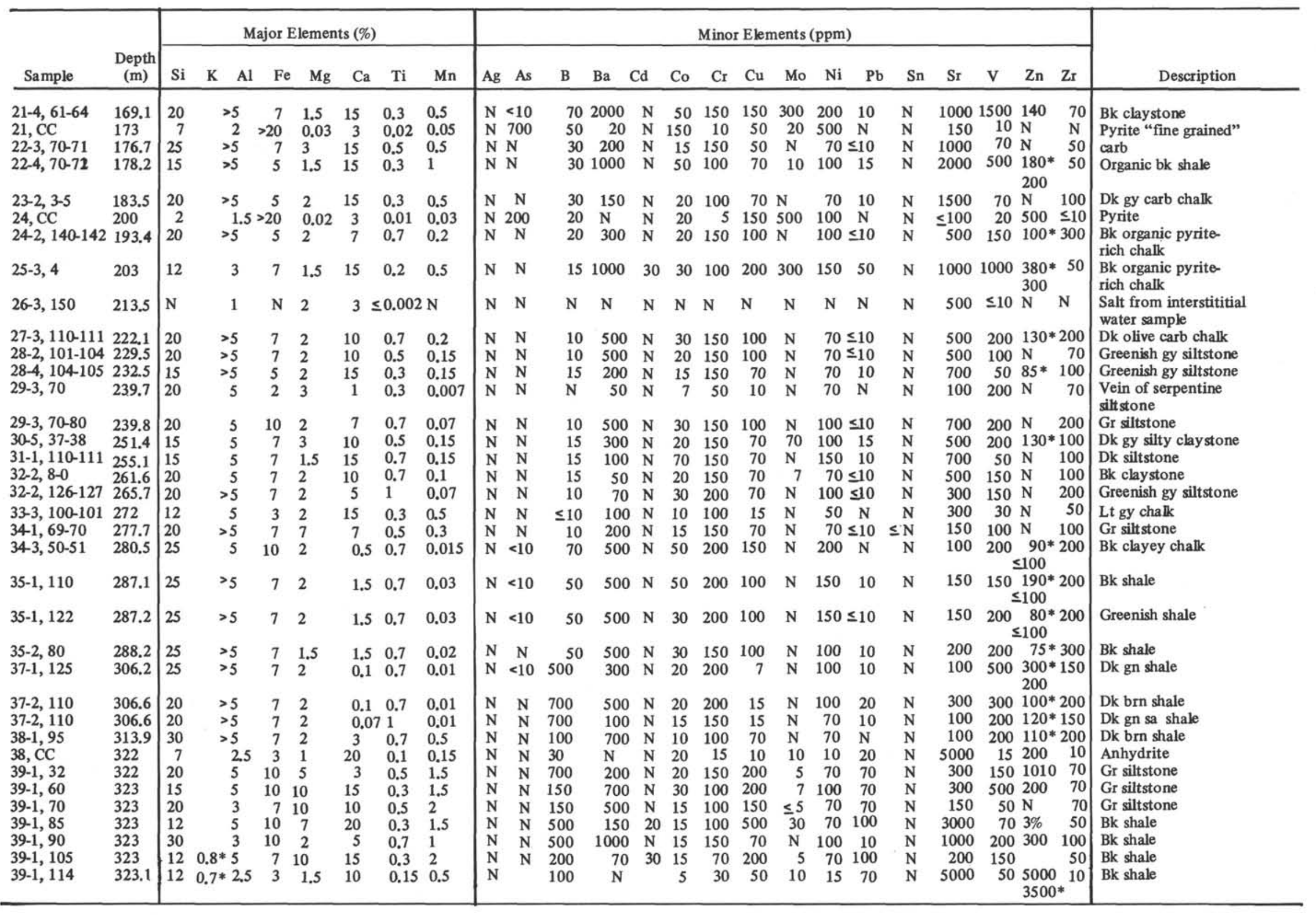


TABLE 4 - Continued

\begin{tabular}{|c|c|c|c|c|c|c|c|c|c|c|c|c|c|c|c|c|c|c|c|c|c|c|c|c|c|c|}
\hline \multirow[b]{2}{*}{ Sample } & \multirow[b]{2}{*}{$\begin{array}{c}\text { Depth } \\
(\mathrm{m})\end{array}$} & \multicolumn{8}{|c|}{ Major Elements (\%) } & \multicolumn{16}{|c|}{ Minor Elements (ppm) } & \multirow[b]{2}{*}{ Description } \\
\hline & & $\mathrm{Si}$ & $\mathrm{K}$ & $\mathrm{Al}$ & $\mathrm{Fe}$ & $\mathrm{Mg}$ & $\mathrm{Ca}$ & $\mathrm{Ti}$ & $\mathrm{Mn}$ & $\mathrm{Ag}$ & As & B & $\mathrm{Ba}$ & Cd & Co & $\mathrm{Cr}$ & $\mathrm{Cu}$ & Mo & $\mathrm{Ni}$ & $\mathrm{Pb}$ & Sn & $\mathrm{Sr}$ & V & $\mathrm{Zn}$ & $\mathrm{Lr}$ & \\
\hline $39-1,115$ & 33.1 & 15 & $\begin{array}{l}0.6^{*} \\
1.8^{*}\end{array}$ & 5 & 15 & 5 & 0.5 & 0.2 & 1.5 & $\mathrm{~N}$ & 10 & 200 & 30 & $\mathrm{~N}$ & 20 & 000 & 100 & 70 & 100 & 100 & $\mathrm{~N}$ & 2000 & 100 & $\begin{array}{l}1300^{*} \\
2000\end{array}$ & 20 & Bk shale \\
\hline $39-1,115$ & 323 & 20 & & 3 & 10 & 5 & 3 & 0.5 & 1 & $\mathrm{~N}$ & $\mathrm{~N}$ & 700 & 200 & $\mathrm{~N}$ & 50 & 100 & 300 & 7 & 150 & 100 & $\mathrm{~N}$ & 200 & 200 & 100 & 100 & Bk shale \\
\hline $39-1,122$ & 323 & 20 & & 5 & 7 & 5 & 5 & 0.5 & 1 & $\mathrm{~N}$ & $\mathrm{~N}$ & 300 & 300 & $\mathrm{~N}$ & 10 & 50 & 150 & $\leq 5$ & 50 & 50 & $\mathrm{~N}$ & 200 & 100 & $\mathrm{~N}$ & 150 & Gr siltstone \\
\hline $39-1,126$ & 323 & 20 & & 5 & 10 & 5 & 3 & 0.5 & 1.5 & $\mathrm{~N}$ & $\mathrm{~N}$ & 700 & 500 & $\mathrm{~N}$ & 20 & 150 & 200 & $\leq 5$ & 100 & 50 & $\mathrm{~N}$ & 150 & 200 & 300 & 100 & Gr siltstone \\
\hline $39-1,130$ & 323 & 15 & & 5 & 10 & 5 & 5 & 0.3 & 3 & $\mathrm{~N}$ & $\mathrm{~N}$ & 500 & 500 & $\mathrm{~N}$ & 20 & 70 & 200 & $\leq 5$ & 50 & 70 & $\mathrm{~N}$ & 150 & 100 & 300 & 70 & Gr siltstone \\
\hline $39-1,140$ & 323 & 20 & & 5 & 7 & 7 & 7 & 0.7 & 1 & $\mathrm{~N}$ & $\mathrm{~N}$ & 700 & 300 & L & 20 & 150 & 150 & $\leq 5$ & 70 & 70 & $\mathrm{~N}$ & 1500 & 200 & 1500 & 150 & Gr siltstone \\
\hline $39-1,145$ & 323 & 20 & & 5 & 7 & 5 & 3 & 0.5 & 0.5 & $\mathrm{~N}$ & $\mathrm{~N}$ & 500 & 500 & $\mathrm{~L}$ & 15 & 100 & 150 & $\leq 5$ & 70 & 30 & $\mathrm{~N}$ & 1500 & 150 & 1000 & 70 & Gr siltstone \\
\hline
\end{tabular}

Note:

See Table 1.A for explanation of abbreviations. 
TABLE 5

Semiquantitative Spectrographic Analyses of Samples from Site 229

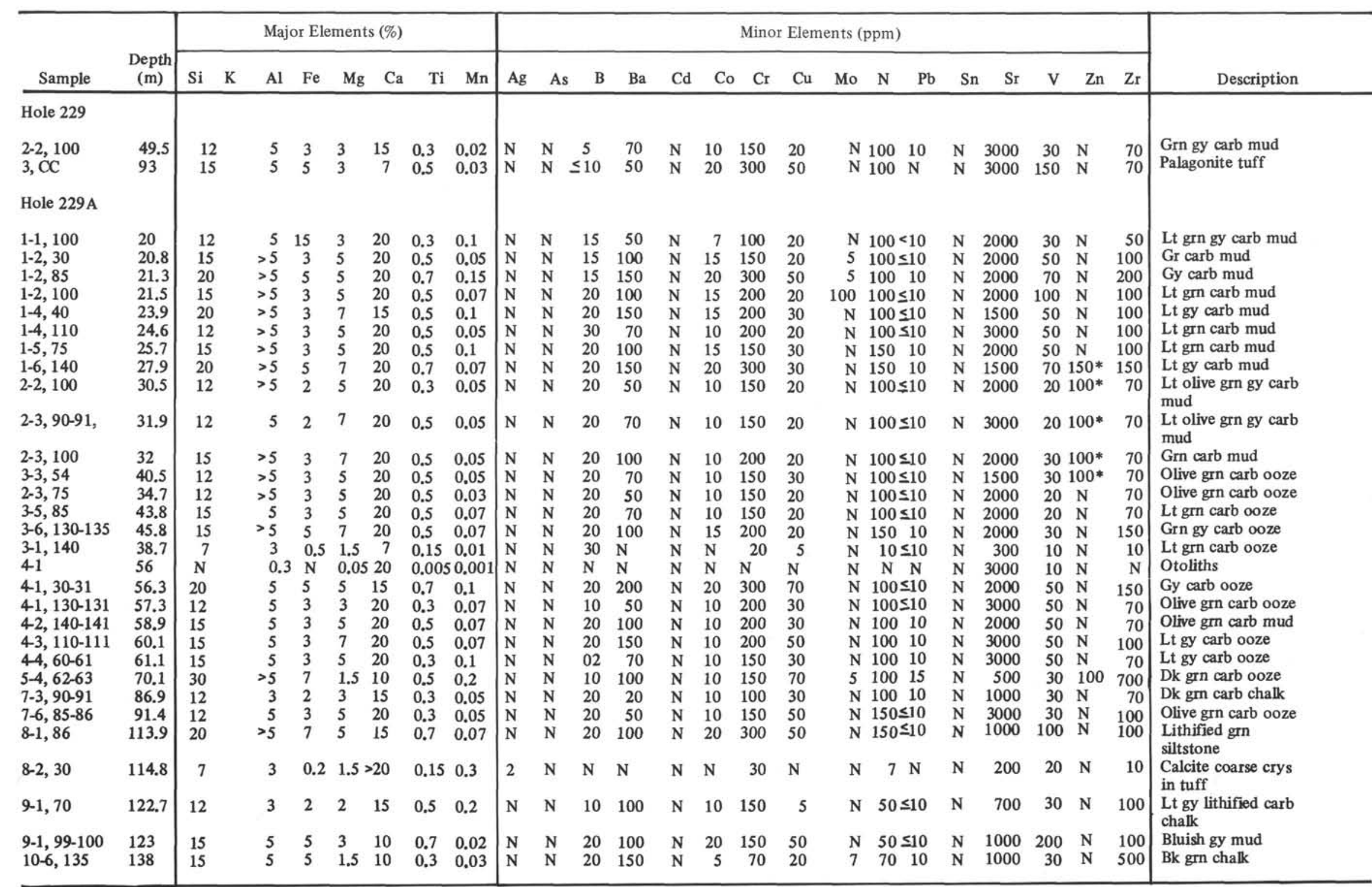


F. MANHEIM, D. SIEMS

TABLE 6

Major Element Analysis of Sediments from Selected Leg 23 Sediments

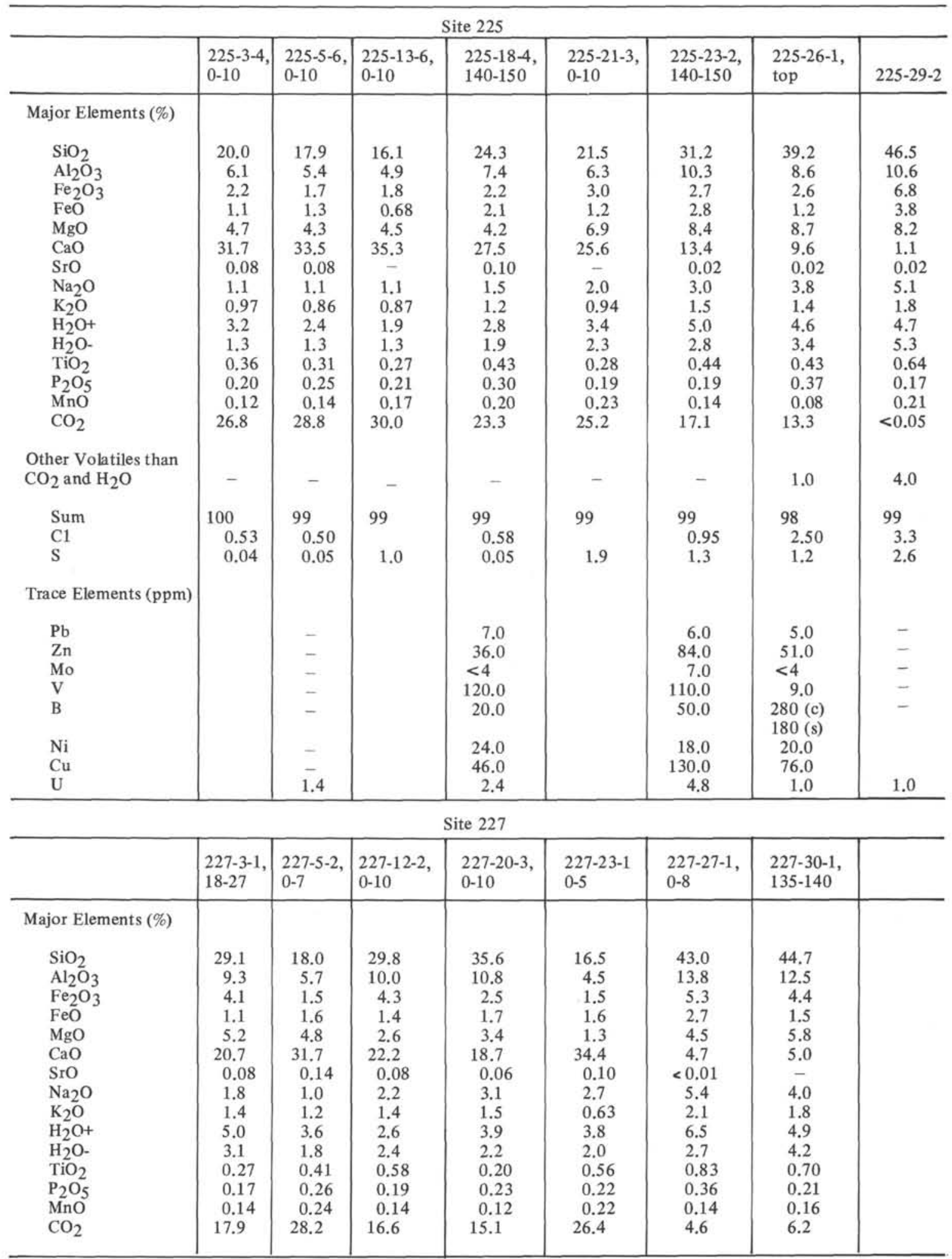


TABLE 6 - Continued

\begin{tabular}{|c|c|c|c|c|c|c|c|c|}
\hline \multicolumn{9}{|c|}{ Site 227 (Cont) } \\
\hline & $\begin{array}{l}227-3-1 \\
18-27\end{array}$ & $\begin{array}{l}227-5-2 \\
0-7\end{array}$ & $\begin{array}{l}227-12-2 \\
0-10\end{array}$ & $\begin{array}{l}227-20-3 \\
0-10\end{array}$ & $\begin{array}{l}227-23-1, \\
0-5\end{array}$ & $\begin{array}{l}227-27-1, \\
0-8\end{array}$ & $\begin{array}{l}227-30-1 \\
135-140\end{array}$ & \\
\hline $\begin{array}{l}\text { Other Volatiles than } \\
\mathrm{CO}_{2} \text { and } \mathrm{H}_{2} \mathrm{O}\end{array}$ & - & - & - & - & 1.6 & - & - & \\
\hline Sum & 99 & 100 & 96 & 99 & 98 & 97 & 96 & \\
\hline $\mathrm{Cl}$ & 0.65 & 0.60 & 1.05 & 1.95 & 2.0 & 2.0 & & \\
\hline $\mathrm{S}$ & 1.7 & 0.04 & 2.2 & 0.23 & 0.84 & 2.2 & 2.9 & \\
\hline \multicolumn{9}{|l|}{ Trace Elements (ppm) } \\
\hline $\mathrm{Pb}$ & - & - & - & - & 3.0 & 5.0 & 5.0 & \\
\hline $\mathrm{Zn}$ & - & - & - & - & 24.0 & 49.0 & 35.0 & \\
\hline $\mathrm{Cu}$ & - & - & - & - & 58.0 & 220.0 & 48.0 & \\
\hline $\mathrm{Ni}$ & - & - & - & - & 28.0 & 38.0 & 44.0 & \\
\hline Mo & - & - & - & - & 28.0 & 4.0 & $<4.0$ & \\
\hline V & - & - & - & - & 150.0 & 240.0 & 100.0 & \\
\hline \multirow[t]{2}{*}{ B } & - & - & - & - & $<20(s)$ & $70(s)$ & 500 & \\
\hline & - & - & - & - & $51(\mathrm{c})$ & $96(\mathrm{c})$ & - & \\
\hline $\mathrm{U}$ & 2.2 & 6.4 & 1.4 & 8.8 & 4.4 & - & - & \\
\hline \multicolumn{9}{|c|}{ Site 228} \\
\hline & $\begin{array}{l}228-5-3 \\
0-10\end{array}$ & $\begin{array}{l}228-10-6 \\
0-10\end{array}$ & $\begin{array}{l}228-16-5 \\
0-10\end{array}$ & $\begin{array}{l}228-24-3 \\
140-150\end{array}$ & $228-30$ & $\begin{array}{l}228-33-1, \\
\text { top }\end{array}$ & $\begin{array}{l}228-35-1 \\
140 / 150\end{array}$ & 228-39, CC. \\
\hline \multicolumn{9}{|l|}{ Major Elements (\%) } \\
\hline $\mathrm{SiO}_{2}$ & 38.4 & 46.3 & 38.2 & 46.1 & 50.4 & 50.6 & 56.4 & 35.3 \\
\hline $\mathrm{Al}_{2} \mathrm{O}_{3}$ & 10.3 & 13.3 & 10.9 & 13.5 & 15.7 & 16.6 & 15.6 & 9.6 \\
\hline $\mathrm{Fe}_{2} \mathrm{O}_{3}$ & 3.6 & 4.6 & 3.8 & 4.1 & 4.4 & 4.1 & 3.5 & 3.3 \\
\hline $\mathrm{FeO}$ & 1.5 & 2.2 & 2.5 & 2.0 & 2.6 & 2.7 & 2.8 & 2.3 \\
\hline $\mathrm{MgO}$ & 3.0 & 3.4 & 3.0 & 3.0 & 3.3 & 3.1 & 3.1 & 3.0 \\
\hline $\mathrm{CaO}$ & 19.9 & 11.1 & 17.9 & 11.5 & 6.0 & 4.5 & 1.4 & 14.5 \\
\hline SrO & 0.08 & & 0.08 & 0.06 & 0.05 & 0.04 & 0.01 & 0.12 \\
\hline $\mathrm{Na}_{2} \mathrm{O}$ & 2.2 & 2.7 & 2.8 & 3.0 & 3.2 & 3.2 & 4.2 & 2.0 \\
\hline $\mathrm{K}_{2} \mathrm{O}$ & 1.3 & 1.6 & 1.4 & 1.8 & 2.2 & 2.2 & 2.3 & 2.0 \\
\hline $\mathrm{H}_{2} \mathrm{O}+$ & 2.8 & 4.4 & 3.4 & 4.2 & 5.0 & 6.0 & 5.0 & 3.5 \\
\hline $\mathrm{H}_{2} \mathrm{O}$ & 1.1 & 1.5 & 1.3 & 1.2 & 1.9 & 2.4 & 2.2 & 1.0 \\
\hline $\mathrm{TiO}_{2}$ & 0.90 & 0.71 & 0.81 & 0.87 & 0.98 & 0.92 & 1.0 & 0.66 \\
\hline $\mathrm{P}_{2} \mathrm{O} 5$ & 0.24 & 0.25 & 0.27 & 0.22 & 0.24 & 0.18 & 0.18 & 0.15 \\
\hline $\mathrm{MnO}$ & 0.11 & 0.16 & 0.21 & 0.16 & 0.14 & 0.06 & 0.06 & 0.43 \\
\hline $\mathrm{CO}_{2}$ & 13.9 & 7.0 & 12.5 & 7.7 & 3.6 & 2.4 & $<0.05$ & 2.8 \\
\hline \multicolumn{9}{|l|}{ Other Volatiles than } \\
\hline $\mathrm{CO}_{2}$ and $\mathrm{H}_{2} \mathrm{O}$ & - & - & - & - & - & - & 2.0 & 1.0 \\
\hline Sum & 99 & 99 & 99 & 99 & 100 & 99 & 100 & 82 \\
\hline $\mathrm{Cl}$ & 0.28 & 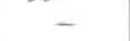 & 0.70 & 0.65 & 0.66 & 0.62 & 1.35 & 1.10 \\
\hline $\mathrm{S}$ & 0.00 & 0.21 & 0.13 & 0.07 & 0.20 & 0.50 & 0.88 & 8.2 \\
\hline \multicolumn{9}{|l|}{ Trace Elements (ppm) } \\
\hline $\mathrm{Pb}$ & - & 6.0 & - & 5.0 & - & 5.0 & 6.0 & 14.0 \\
\hline $\mathrm{Zn}$ & - & 56.0 & - & 65.0 & - & 85.0 & 180.0 & 540.0 \\
\hline $\mathrm{Cu}$ & - & 52.0 & - & 38.0 & 48.0 & 53.0 & 46.0 & 33.0 \\
\hline Mo & - & 38.0 & - & $<4$ & $<4$ & $<4$ & $<4$ & $<4$ \\
\hline V & - & $<4$ & - & 120.0 & 140.0 & 120.0 & 110.0 & 90.0 \\
\hline $\mathrm{Ni}$ & - & 200.0 & - & 28.0 & 42.0 & 38.0 & 22.0 & 15.0 \\
\hline \multirow[t]{2}{*}{ B } & - & 40.0 & - & $40(\mathrm{~s})$ & $40(s)$ & $50(s)$ & $40(s)$ & $110(\mathrm{~s})$ \\
\hline & - & - & - & $41(\mathrm{c})$ & $24(\mathrm{c})$ & $100(c)$ & & \\
\hline $\mathrm{U}$ & 1.6 & - & - & 1.2 & 2.4 & 2.4 & 1.6 & 1.2 \\
\hline
\end{tabular}


TABLE 6 - Continued

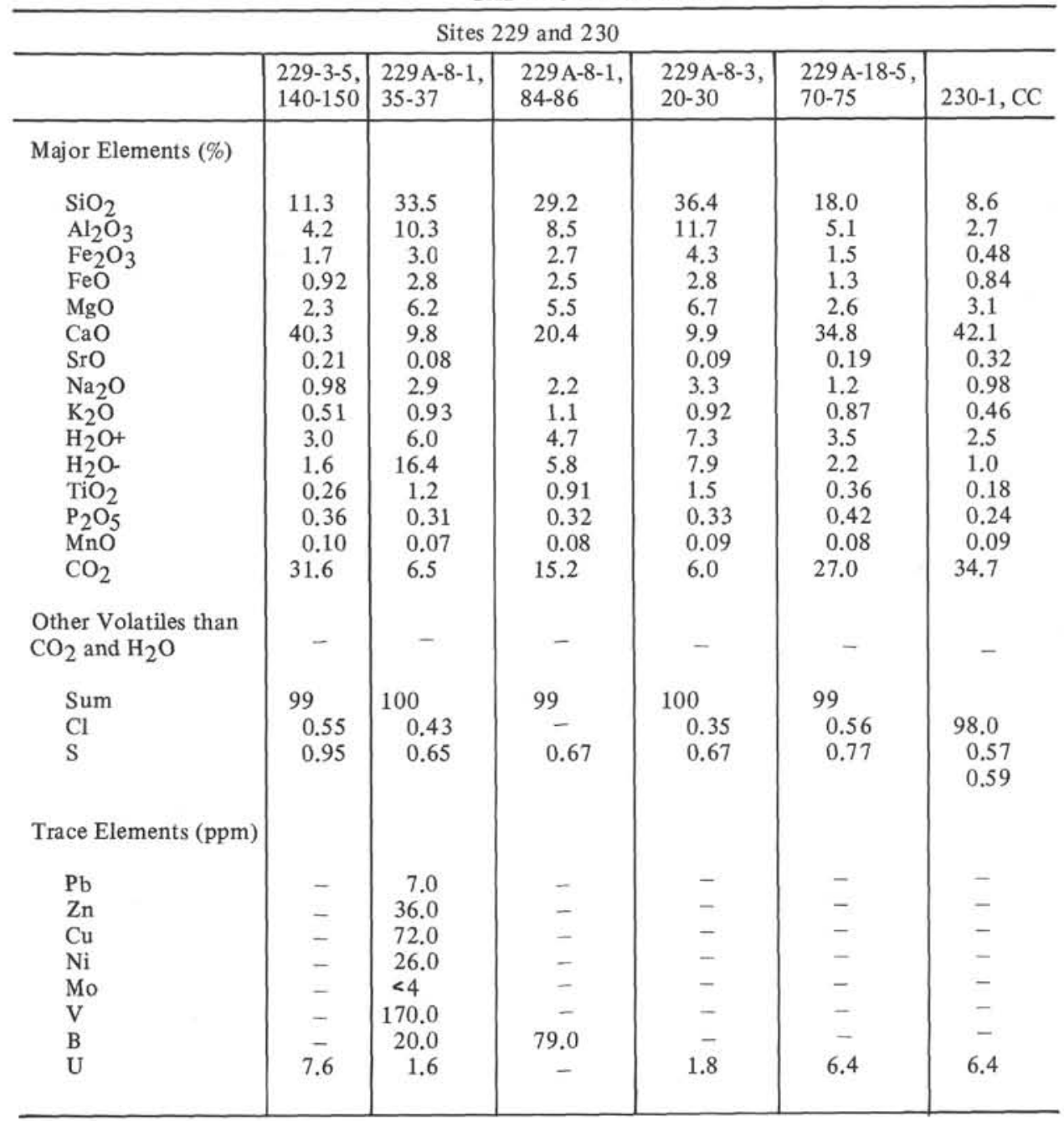

Note:

"Rapid rock analyses" performed by the U.S. G.S., Washington, Leonard Shapiro, Supervising analyst. Quantitative spectrochemical analyses by laboratory under leadership of C. Annell; supplementary analyses $(\mathrm{Cl}, \mathrm{SrO}, \mathrm{B}, \mathrm{U}$, by wet chemical laboratory under leadership of J.I. Dinnin). 